\title{
commuting q-analogue of the addition formula for disk polynomials
}

\author{
elink, H.T.; Floris, P.G.A. \\ $1007 / s 003659900057$ \\ blication date \\ 97 \\ blished in \\ nstructive Approximation
}

$\mathrm{k}$ to publication

ation for published version (APA):

elink, H. T., \& Floris, P. G. A. (1997). A commuting q-analogue of the addition formula for k polynomials. Constructive Approximation, 13, 511-535.

os://doi.org/10.1007/s003659900057

\section{neral rights}

not permitted to download or to forward/distribute the text or part of it without the consent of the author(s) //or copyright holder(s), other than for strictly personal, individual use, unless the work is under an open tent license (like Creative Commons). 


\title{
A Commuting $q$-Analogue of the Addition Formula for Disk Polynomials
}

\author{
P. G. A. Floris and H. T. Koelink
}

\begin{abstract}
Starting from the addition formula for $q$-disk polynomials, which is an identity in noncommuting variables, we establish a basic analogue in commuting variables of the addition and product formula for disk polynomials. These contain, as limiting cases, the addition and product formula for little $q$-Legendre polynomials. As $q$ tends to 1 the addition and product formula for disk polynomials are recovered.
\end{abstract}

\section{Introduction}

It is known that there is a strong connection between the theories of hypergeometric functions and group representations. The identification of certain families of hypergeometric functions as specific functions on groups enabled proofs of new identities as well as alternative, more conceptual, proofs of well-known identities for these families. Particular examples of such identities are so-called addition formulas. From the group-theoretic point of view addition formulas reflect the homomorphism property of group representations; for details we refer the reader to Vilenkin [18] and Vilenkin and Klimyk [19].

$q$-Special functions are generalizations (or deformations) of classical special functions, having comparable properties, and were first studied in the eighteenth and nineteenth centuries by Euler, Gauss, Heine, and others. And although a lot is known about these functions, especially the ones of basic hypergeometric type, there has for a long time been no satisfactory mathematical structure on which they appear as naturally as hypergeometric functions do on groups. The introduction of quantum groups in the 1980s seems to have met the need for such structures. It has now been widely accepted that quantum groups (and quantized algebras) form a natural setting for many of the well-known families of basic hypergeometric functions. Of particular interest is the realization of Askey-Wilson polynomials, at least for specific choices of the parameters, in quantum group theory; see Koornwinder [11], Noumi and Mimachi [12], Koelink [7], Dijkhuizen and Noumi [3], and references given there.

The very nature of the objects one is dealing with in this area accounts for the appearance of identities in noncommuting variables, or, better, in variables that satisfy certain

Date received: September 29, 1995. Date revised: May 20, 1996. Communicated by Mourad Ismail. AMS classification: 33D45, 33D80.

Key words and phrases: $q$-Disk polynomials, Little $q$-Jacobi polynomials, Affine $q$-Krawtchouk polynomials, Addition formula, Product formula. 
explicit commutation relations. Such identities are of interest in their own right and are perhaps the natural ones to expect. However, it is legitimate to ask whether it is possible to convert such an identity into one involving only commuting variables without losing any information. A way to do this is to represent the identity under consideration as an operator identity in some Hilbert space and then to construct matrix elements, depending on certain parameters, to obtain a scalar identity involving these additional parameters. It is this procedure that we use in this paper to obtain a commuting $q$-analogue of the addition formula for disk polynomials.

Disk polynomials are polynomials $R_{l, m}^{(v)}(z)\left(l, m \in \mathbb{Z}_{+}, v>-1\right)$ in the two variables $z$ and $\bar{z}$ that are orthogonal with respect to a positive measure on the closed unit disk $D$ in the complex plane. Explicitly, they are given as

$$
R_{l, m}^{(\nu)}(z)= \begin{cases}z^{l-m} P_{m}^{(v, l-m)}\left(2|z|^{2}-1\right) & (l \geq m), \\ \bar{z}^{m-l} P_{l}^{(v, m-l)}\left(2|z|^{2}-1\right) & (l \leq m),\end{cases}
$$

where the $P_{k}^{(\alpha, \beta)}(x)$ denotes the Jacobi polynomial. Their orthogonality reads

$$
\iint_{D} R_{l, m}^{(v)}\left(r e^{i \phi}\right) \overline{R_{l^{\prime}, m^{\prime}}^{(v)}\left(r e^{i \phi}\right)} r\left(1-r^{2}\right)^{v} d r d \phi=0 \quad \text { if } \quad(l, m) \neq\left(l^{\prime}, m^{\prime}\right) .
$$

For integer values $v=n-2$ the disk polynomials are the zonal spherical functions on the compact homogeneous space $U(n) / U(n-1)$, which can be identified with the unit sphere in $\mathbb{C}^{n}$. Here $U(n)$ denotes the group of unitary transformations of $\mathbb{C}^{n}$. An addition formula for these polynomials was proved independently by S̆apiro [14] and Koornwinder [9] around 1970. For $v>0$ it takes the following form

$$
\begin{aligned}
& R_{l, m}^{(v)}\left(\cos \theta_{1} e^{i \phi_{1}} \cos \theta_{2} e^{i \phi_{2}}+\sin \theta_{1} \sin \theta_{2} \rho e^{i \psi}\right) \\
& =\sum_{r=0}^{l} \sum_{s=0}^{m} C_{l, m ; r, s}^{(v)}\left(\sin \theta_{1}\right)^{r+s} R_{l-r, m-s}^{(v+r+s)}\left(\cos \theta_{1} e^{i \phi_{1}}\right) \\
& \quad \times\left(\sin \theta_{2}\right)^{r+s} R_{l-r, m-s}^{(v+r+s)}\left(\cos \theta_{2} e^{i \phi_{2}}\right) R_{r, s}^{(\nu-1)}\left(\rho e^{i \psi}\right),
\end{aligned}
$$

where

$$
C_{l, m ; r, s}^{(v)}=\frac{v}{v+r+s}\left(\begin{array}{l}
l \\
r
\end{array}\right)\left(\begin{array}{c}
m \\
s
\end{array}\right) \frac{(v+l+1)_{s}(v+m+1)_{r}}{(v+r)_{s}(v+s)_{r}} .
$$

The corresponding product formula, which follows from (1.3) and (1.2), is given by

$$
\begin{aligned}
& R_{l, m}^{(v)}\left(\cos \theta_{1} e^{i \phi_{1}}\right) R_{l, m}^{(\nu)}\left(\cos \theta_{2} e^{i \phi_{2}}\right) \\
& =\frac{\nu}{\pi} \int_{0}^{1} \int_{0}^{2 \pi} R_{l, m}^{(\nu)}\left(\cos \theta_{1} e^{i \phi_{1}} \cos \theta_{2} e^{i \phi_{2}}+\sin \theta_{1} \sin \theta_{2} r e^{i \psi}\right) r\left(1-r^{2}\right)^{\nu-1} d \psi d r .
\end{aligned}
$$

The addition formula for Jacobi polynomials can be obtained from the addition formula (1.3) for disk polynomials, see [9] and [19, Vol. 2, §11.4.2].

A quantum analogue of the homogeneous space $U(n) / U(n-1)$ has been studied by Noumi, Yamada, and Mimachi [13]; see also Floris [4]. They derive the following expression for the zonal spherical elements in terms of little $q$-Jacobi polynomials $p_{k}\left(x ; q^{\alpha}, q^{\beta} ; q\right)$ defined in $(2.3)$

$$
R_{l, m}^{(v)}\left(z, z^{*} ; q^{2}\right)= \begin{cases}z^{l-m} p_{m}\left(1-z z^{*} ; q^{2 v}, q^{2 l-2 m} ; q^{2}\right) & (l \geq m), \\ p_{l}\left(1-z z^{*} ; q^{2 v}, q^{2 m-2 l} ; q^{2}\right)\left(z^{*}\right)^{m-l} & (l \leq m),\end{cases}
$$


where $v=n-2$ and $z$ and $z^{*}$ are the generators of a complex $*$-algebra, satisfying the relation $z^{*} z=q^{2} z z^{*}+1-q^{2}$. Here the deformation parameter $q$ is assumed to be such that $0<q<1$. The polynomials of (1.5) are now called $q$-disk polynomials. In the paper [4] an addition theorem for the $q$-disk polynomials is proved, which is reproduced in Section 2. It is an identity in several noncommuting variables which has a structure similar to (1.3). It is this addition formula that we convert into one involving only commuting variables, using the procedure mentioned before. The outcome of it is the basic analogue of the classical addition formula for disk polynomials and it is the main result of this paper.

The remaining sections are organized as follows. In Section 2 we recall some facts on $q$-disk polynomials needed later on. In particular, we state the addition formula. Section 3 deals with irreducible $*$-representations of the $*$-algebras on which the addition formula is realized. This information is used in Section 4 to represent the addition formula as an operator identity on some infinite-dimensional Hilbert space. Moreover, we give the eigenspace decomposition of this Hilbert space with respect to a specific self-adjoint operator that appears in the left-hand side of the addition formula under such an irreducible $*$-representation. The eigenvectors are expressible in terms of affine $q$-Krawtchouk polynomials, and we write down explicitly the action of the left-hand side on these eigenvectors. In Section 5 we calculate the image of the standard basis under the action of the right-hand side of the operator identity. The commutative addition formula is obtained from the fact that we use $*$-representations. In Section 6 a corresponding product formula is derived. Finally, Section 7 discusses the limit transitions $q \rightarrow 1$ of both the addition and the product formula to the classical identities (1.3) and (1.4).

The notation for basic hypergeometric series follows the book [5] by Gasper and Rahman. By $\mathbb{Z}_{+}$we denote $\{0,1,2, \ldots\}$ and $a \wedge b=\min (a, b)$ for $a, b \in \mathbb{R}$. Throughout this paper we fix $0<q<1$.

\section{Addition Formula for $q$-Disk Polynomials}

In [4] an addition formula for the $q$-analogue of the disk polynomials defined in (1.5) has been derived. In this section we recall this result in a slightly simpler version. In order to present the addition formula we first introduce certain noncommutative algebras and the little $q$-Jacobi polynomials that play a role in this identity.

First, we introduce the $*$-algebra $\widetilde{\mathcal{X}}$, which is the quotient of the $*$-algebra $\mathcal{X}$ of $[4$, $\S 4.5]$ by the ideal generated by $Q-1$. So $\widetilde{\mathcal{X}}$ is generated by $X_{1}, X_{2}$ subject to the relations

$$
\begin{gathered}
X_{1} X_{2}=q X_{2} X_{1}, \quad X_{1}^{*} X_{2}=q X_{2} X_{1}^{*}, \quad X_{2}^{*} X_{2}=q^{2} X_{2} X_{2}^{*}+1-q^{2}, \\
X_{1}^{*} X_{1}=q^{2} X_{1} X_{1}^{*}+\left(1-q^{2}\right)\left(1-X_{2} X_{2}^{*}\right) .
\end{gathered}
$$

We use the same notation for the generators of $\tilde{\mathcal{X}}$ and $\mathcal{X}$.

We also introduce the $*$-algebra $\widetilde{\mathcal{Y}}$, which is the quotient of the $*$-algebra $\mathcal{Y}$ of $[4$, $\S 4.5]$ by the ideal generated by $D-1$. So $\widetilde{\mathcal{Y}}$ is generated by $Y_{1}, Y_{2}$ subject to the relations

$$
\begin{gathered}
Y_{1} Y_{2}=q Y_{2} Y_{1}, \quad Y_{1}^{*} Y_{2}=q Y_{2} Y_{1}^{*}, \quad Y_{1}^{*} Y_{1}=Y_{1} Y_{1}^{*}, \\
Y_{1} Y_{1}^{*}+Y_{2} Y_{2}^{*}=1=q^{2} Y_{1}^{*} Y_{1}+Y_{2}^{*} Y_{2} .
\end{gathered}
$$


Also, here we use the same notation for the generators of $\tilde{\mathcal{Y}}$ and $\mathcal{Y}$. These $*$-algebras are closely related to the deformed $*$-algebra of polynomials on the sphere in $\mathbb{C}^{n}$ for $n \geq 3$ in the case of $\widetilde{\mathcal{X}}$ and for $n=2$ in the case of $\widetilde{\mathcal{Y}}$, see $[4, \S 4.5]$.

The little $q$-Jacobi polynomials are defined by

$$
p_{l}(x ; a, b ; q)={ }_{2} \varphi_{1}\left(\begin{array}{c}
q^{-l}, a b q^{l+1} \\
a q
\end{array} ; q, q x\right) .
$$

These polynomials are orthogonal polynomials on a geometric sequence, see [1], [5], and references therein. Using the little $q$-Jacobi polynomials we define

$$
R_{l, m}^{(v)}\left(x, y, z ; q^{2}\right)= \begin{cases}z^{m} x^{l-m} p_{m}\left((z-x y) z^{-1} ; q^{2 v}, q^{2(l-m)} ; q^{2}\right) & (l \geq m), \\ z^{l} p_{l}\left((z-x y) z^{-1} ; q^{2 v}, q^{2(m-l)} ; q^{2}\right) y^{m-l} & (l \leq m),\end{cases}
$$

which extends the definition (1.5). We use (2.4) for noncommuting variables $x, y, z$, with $y x=q^{2} x y+\left(1-q^{2}\right) z$ where we assume that $z$ commutes with $x$ and $y$, so that (2.4) is polynomial in $x, y$, and $z$.

The following addition theorem follows directly from [4, Theorem 4.30] with $Q=1$, $D=1$.

Theorem 2.1. The following addition formula holds as an identity in $\tilde{\mathcal{X}} \otimes \tilde{\mathcal{Y}}$ for $v>0$ :

$$
\begin{aligned}
& R_{l, m}^{(\nu)}\left(-q X_{1} \otimes Y_{1}^{*}+X_{2} \otimes Y_{2},-q X_{1}^{*} \otimes Y_{1}+X_{2}^{*} \otimes Y_{2}^{*}, 1 \otimes 1 ; q^{2}\right) \\
& =\sum_{r=0}^{l} \sum_{s=0}^{m} c_{l, m ; r, s}^{(v)}\left(q^{2}\right) R_{l-r, m-s}^{(\nu+r+s)}\left(X_{2}, X_{2}^{*}, 1 ; q^{2}\right) R_{r, s}^{(\nu-1)}\left(X_{1}, X_{1}^{*}, 1-X_{2} X_{2}^{*} ; q^{2}\right) \\
& \quad \otimes(-q)^{r-s} R_{l-r, m-s}^{(v+r+s)}\left(Y_{2}, Y_{2}^{*}, 1 ; q^{2}\right) Y_{1}^{s}\left(Y_{1}^{*}\right)^{r},
\end{aligned}
$$

with

$$
\begin{aligned}
c_{l, m ; r, s}^{(\nu)}(q) & =\frac{1-q^{\nu+r+s+1}}{1-q^{\nu+1}} \frac{c_{l, m}^{(\nu)}(q)}{c_{l-r, m-s}^{(v+r+s)}(q) c_{r, s}^{(\nu-1)}(q)}, \\
c_{l, m}^{(v)}(q) & =\frac{q^{m(v+1)}\left(1-q^{\nu+1}\right)}{1-q^{\nu+l+m+1}} \frac{(q ; q)_{l}(q ; q)_{m}}{\left(q^{\nu+1} ; q\right)_{l}\left(q^{\nu+1} ; q\right)_{m}} .
\end{aligned}
$$

The proof of Theorem 2.1 is based on the interpretation of $q$-disk polynomials for $v=n-2$ as zonal spherical functions on a quantum analogue of the homogeneous space $U(n) / U(n-1)$; see also [13]. The addition formula is essentially the development in terms of associated spherical functions of the outcome of the comultiplication applied to such a spherical element.

The main goal of this paper is to deduce an addition formula in commuting variables from $(2.5)$ using $*$-representations of the $*$-algebras $\widetilde{\mathcal{X}}$ and $\widetilde{\mathcal{Y}}$. These $*$-representations are studied in the next section.

\section{Some Representation Theory}

In this section we study $*$-representations of $\tilde{\mathcal{X}}$ and $\tilde{\mathcal{Y}}$ using the classification of the irreducible $*$-representations of the Hopf $*$-algebra of polynomials on the quantum $U(n)$ 
group for $n=2,3$. This Hopf $*$-algebra is denoted by $\operatorname{Pol}\left(U_{q}(n)\right)$ in $[6, \S 2]$, by $\mathcal{A}_{q}(n)$ in $[4, \S 3.2]$, and by $A\left(U_{q}(n)\right)$ in $[13, \S 3.1]$. We stick to the notation $\operatorname{Pol}\left(U_{q}(n)\right)$ of [6], and we also use the notation $\operatorname{Pol}\left(S U_{q}(n)\right)$ for the Hopf $*$-algebra which is a quotient of $\operatorname{Pol}\left(U_{q}(n)\right)$ by the ideal generated by $D-1$. Here $D \in \operatorname{Pol}\left(U_{q}(n)\right)$ is the quantum determinant $[6,(2.7)]$, which is central.

The $*$-algebra $\tilde{\mathcal{Y}}$ is isomorphic to $\operatorname{Pol}\left(S U_{q}(2)\right)$ under the identification

$$
\left(\begin{array}{ll}
t_{11} & t_{12} \\
t_{21} & t_{22}
\end{array}\right)=\left(\begin{array}{cc}
Y_{2}^{*} & -q Y_{1}^{*} \\
Y_{1} & Y_{2}
\end{array}\right),
$$

where $t_{i j}, 1 \leq i, j \leq 2$, are the generators of $\operatorname{Pol}\left(S U_{q}(2)\right)$ satisfying the commutation relations of $[6, \S 2]$ with $D=1$. The irreducible $*$-representations of this algebra have been classified by Vaksman and Soibelman [16, Theorem 3.2]; see also [6, Theorem 4.8].

Proposition 3.1. All mutually inequivalent irreducible $*$-representations of $\tilde{\mathcal{Y}}$ are

(1) one-dimensional $*$-representations for $\phi \in[0,2 \pi)$,

$$
Y_{1} \longmapsto 0, \quad Y_{2} \longmapsto e^{i \phi} ;
$$

(2) infinite-dimensional $*$-representations $\sigma_{\phi}, \phi \in[0,2 \pi)$, acting in $\ell^{2}\left(\mathbb{Z}_{+}\right)$with orthonormal basis $\left\{e_{n} \mid n \in \mathbb{Z}_{+}\right\}$,

$$
\begin{aligned}
\sigma_{\phi}\left(Y_{1}\right) e_{n} & =e^{i \phi} q^{n} e_{n}, & \sigma_{\phi}\left(Y_{2}\right) e_{n} & =\sqrt{1-q^{2 n+2}} e_{n+1}, \\
\sigma_{\phi}\left(Y_{1}^{*}\right) e_{n} & =e^{-i \phi} q^{n} e_{n}, & & \sigma_{\phi}\left(Y_{2}^{*}\right) e_{n}=\sqrt{1-q^{2 n}} e_{n-1},
\end{aligned}
$$

where $e_{n}=0$ for negative $n$ by convention.

Remark 3.2. Although $\sigma_{\phi}$ is not faithful, it is known that $\bigcap_{\phi} \operatorname{ker}\left(\sigma_{\phi}\right)$ is trivial.

To describe irreducible $*$-representations of $\widetilde{\mathcal{X}}$ suited for our purposes we use the embedding $\widetilde{\mathcal{X}} \hookrightarrow \operatorname{Pol}\left(U_{q}(3)\right)$ as $*$-algebras given by

$$
X_{1} \longmapsto t_{21}^{*}, \quad X_{2} \longmapsto t_{11}^{*} .
$$

The commutation relations (2.1) for $\tilde{\mathcal{X}}$ correspond to [6, (2.1), (2.14), (2.16)]. Note that (3.1) also gives an embedding $\widetilde{\mathcal{X}} \hookrightarrow \operatorname{Pol}\left(U_{q}(n)\right)$ for $n \geq 3$ (this embedding also follows from a combination of [4, Proposition 4.28] with [4, Proposition 4.5] and the fact that the map $t_{i j} \rightarrow t_{n-j+1, n-i+1}$ extends to an algebra antiautomorphism of $\left.\operatorname{Pol}\left(U_{q}(n)\right)\right)$. The irreducible $*$-representations of $\operatorname{Pol}\left(U_{q}(n)\right)$ have been classified in [6, Theorem 4.10], and by specializing $n=3$ and restricting to $\tilde{\mathcal{X}}$ we obtain families of $*$-representations of $\tilde{\mathcal{X}}$. Note that we do not claim the list is exhaustive.

Proposition 3.3. The following list gives mutually inequivalent irreducible *-representations of $\widetilde{\mathcal{X}}$ :

(1) one-dimensional $*$-representations for $\phi \in[0,2 \pi)$

$$
X_{1} \longmapsto 0, \quad X_{2} \longmapsto e^{i \phi} ;
$$


(2) infinite-dimensional $*$-representations $\pi_{1}^{\phi}, \phi \in[0,2 \pi)$, acting on $\ell^{2}\left(\mathbb{Z}_{+}\right)$with orthonormal basis $\left\{e_{n} \mid n \in \mathbb{Z}_{+}\right\}$;

$$
\pi_{1}^{\phi}\left(X_{1}\right) e_{n}=e^{i \phi} q^{n} e_{n}, \quad \pi_{1}^{\phi}\left(X_{2}\right) e_{n}=\sqrt{1-q^{2 n+2}} e_{n+1} ;
$$

(3) infinite-dimensional $*$-representation $\pi$ acting on $\ell^{2}\left(\mathbb{Z}_{+}^{2}\right)$ with orthonormal basis $\left\{f_{\mu} \mid \mu=\left(\mu_{1}, \mu_{2}\right) \in \mathbb{Z}_{+}^{2}\right\}$

$$
\begin{array}{ll}
\pi\left(X_{1}\right) f_{\mu}=q^{\mu_{2}} \sqrt{1-q^{2 \mu_{1}+2}} f_{\mu+\varepsilon_{1}}, & \pi\left(X_{2}\right) f_{\mu}=\sqrt{1-q^{2 \mu_{2}+2}} f_{\mu+\varepsilon_{2}}, \\
\pi\left(X_{1}^{*}\right) f_{\mu}=q^{\mu_{2}} \sqrt{1-q^{2 \mu_{1}}} f_{\mu-\varepsilon_{1}}, & \pi\left(X_{2}^{*}\right) f_{\mu}=\sqrt{1-q^{2 \mu_{2}}} f_{\mu-\varepsilon_{2}},
\end{array}
$$

with $\varepsilon_{1}=(1,0), \varepsilon_{2}=(0,1)$ and the convention that $f_{\mu}=0$ if $\mu_{1}$ or $\mu_{2}$ is negative. This representation is a faithful representation of $\tilde{\mathcal{X}}$.

Proof. Notice that the algebra $\tilde{\mathcal{Y}}$ is isomorphic to the algebra $\widetilde{\mathcal{X}}$ divided out by the ideal generated by $X_{1}^{*} X_{1}-X_{1} X_{1}^{*}$. So parts (1) and (2) follow from Proposition 3.1. It is obvious that the mapping $\pi$ in (3) defines an irreducible $*$-representation of $\widetilde{\mathcal{X}}$ which is not equivalent to the ones in (1) and (2). Hence it remains to prove that $\pi$ is faithful.

Let $\xi \in \widetilde{\mathcal{X}}$ satisfy $\pi(\xi)=0$ and write $\xi$ as a finite sum

$$
\xi=\sum_{r, s, t, u \in \mathbb{Z}_{+}} c_{r, s, t, u} X_{1}^{r}\left(X_{1}^{*}\right)^{s} X_{2}^{t}\left(X_{2}^{*}\right)^{u} .
$$

To simplify calculations we use the orthogonal basis $g_{\mu}=\left(\left(q^{2} ; q^{2}\right)_{\mu_{1}}\left(q^{2} ; q^{2}\right)_{\mu_{2}}\right)^{1 / 2} f_{\mu}$. Then, for all $\mu \in \mathbb{Z}_{+}^{2}$,

$0=\pi(\xi) g_{\mu}=\sum_{r, s, t, u \in \mathbb{Z}_{+}} c_{r, s, t, u}\left(q^{2 \mu_{1}} ; q^{-2}\right)_{s}\left(q^{2 \mu_{2}} ; q^{-2}\right)_{u} q^{\left(\mu_{2}+t-u\right)(s+r)} g_{\mu+(r-s) \varepsilon_{1}+(t-u) \varepsilon_{2}}$.

Consider the coefficient of $g_{\mu+\alpha \varepsilon_{1}+\beta \varepsilon_{2}}$ to find, for fixed $\alpha, \beta$,

$$
\sum_{r=s+\alpha} \sum_{t=u+\beta} c_{r, s, t, u}\left(q^{2 \mu_{1}} ; q^{-2}\right)_{s}\left(q^{2 \mu_{2}} ; q^{-2}\right)_{u} q^{\left(\mu_{2}+t-u\right)(s+r)}=0, \quad \forall \mu_{1}, \mu_{2} \in \mathbb{Z}_{+} .
$$

This double sum is a polynomial in two variables, $q^{\mu_{1}}, q^{\mu_{2}}$, so we find that $c_{r, s, t, u}=0$ for $r=s+\alpha, t=u+\beta$. Since $\alpha, \beta$ are arbitrary, all coefficients are zero. Thus $\xi=0$.

As a consequence of the proof of Proposition 3.3 we obtain

Corollary 3.4. The set $\left\{X_{1}^{r}\left(X_{1}^{*}\right)^{s} X_{2}^{t}\left(X_{2}^{*}\right)^{u} \mid r, s, t, u \in \mathbb{Z}_{+}\right\}$forms a linear basis for $\widetilde{\mathcal{X}}$.

Remark 3.5. Instead of checking the commutation relations in the proof of Proposition 3.3 we can also observe that the actions are coming from the restrictions of certain irreducible *-representations of $\mathrm{Pol}\left(U_{q}(3)\right)$. According to [6, Theorem 4.10] the irreducible *-representations of $\operatorname{Pol}\left(U_{q}(3)\right)$ are parametrized by $\rho \in S_{3}$ and $\left(\gamma_{1}, \gamma_{2}, \gamma_{3}\right) \in \mathbb{C}^{3}$ with $\left|\gamma_{i}\right|$ determined by $\rho$. In case (1) the $*$-representation follows from restriction of the irreducible $*$-representation of $\operatorname{Pol}\left(U_{q}(3)\right)$ corresponding to $\rho=1,\left(\gamma_{1}, \gamma_{2}, \gamma_{3}\right)=$ $\left(e^{-i \phi}, 1,1\right)$; in case (2) we take $\rho=(12),\left(\gamma_{1}, \gamma_{2}, \gamma_{3}\right)=\left(e^{-i \phi},-q, 1\right)$ and in case (3) we take $\rho=(123),\left(\gamma_{1}, \gamma_{2}, \gamma_{3}\right)=(1,-q,-q)$. The orthonormal bases in the cases (2) and (3) correspond to the orthogonal bases described in [6, Corollary 4.15(i)]. 


\section{Spectral Analysis of a Self-Adjoint Operator}

Application of a one-dimensional $*$-representation of either $\tilde{\mathcal{X}}$ or $\tilde{\mathcal{Y}}$, which are described in Section 3, to the addition formula (2.5) leads to a trivial identity, so in order to convert the addition formula (2.5) into an addition formula in commuting variables we apply the infinite-dimensional $\pi \otimes \sigma$ to the identity (2.5) in $\widetilde{\mathcal{X}} \otimes \widetilde{\mathcal{Y}}$, where $\pi$ and $\sigma=\sigma_{0}$ are irreducible $*$-representations of $\widetilde{\mathcal{X}}$ and $\widetilde{\mathcal{Y}}$ introduced in the previous section. In this section we study the operator emerging from the left-hand side of (2.5).

Consider the elements $T, T^{*} \in \widetilde{\mathcal{X}} \otimes \widetilde{\mathcal{Y}}$ defined by

$$
T=-q X_{1} \otimes Y_{1}^{*}+X_{2} \otimes Y_{2}, \quad T^{*}=-q X_{1}^{*} \otimes Y_{1}+X_{2}^{*} \otimes Y_{2}^{*},
$$

and the positive self-adjoint element $R=T T^{*}$. Then the left-hand side of (2.5) is a polynomial in $1-R$ multiplied from the left, respectively, right, by some power of $T$, respectively, $T^{*}$. Here $1=1 \otimes 1$ is the identity of $\widetilde{\mathcal{X}} \otimes \widetilde{\mathcal{Y}}$.

Using Propositions 3.1 and 3.3 we first calculate

and

$$
\begin{aligned}
((\pi \otimes \sigma) T) f_{\mu} \otimes e_{k}= & \sqrt{\left(1-q^{2 \mu_{2}+2}\right)\left(1-q^{2 k+2}\right)} f_{\mu+\varepsilon_{2}} \otimes e_{k+1} \\
& -q^{1+\mu_{2}+k} \sqrt{1-q^{2 \mu_{1}+2}} f_{\mu+\varepsilon_{1}} \otimes e_{k}
\end{aligned}
$$

$$
\begin{aligned}
\left((\pi \otimes \sigma) T^{*}\right) f_{\mu} \otimes e_{k}= & \sqrt{\left(1-q^{2 \mu_{2}}\right)\left(1-q^{2 k}\right)} f_{\mu-\varepsilon_{2}} \otimes e_{k-1} \\
& -q^{1+\mu_{2}+k} \sqrt{1-q^{2 \mu_{1}}} f_{\mu-\varepsilon_{1}} \otimes e_{k}
\end{aligned}
$$

By $H(\mu, p)$ we denote the $(N+1)$-dimensional subspace of the representation space $\ell^{2}\left(\mathbb{Z}_{+}^{2}\right) \otimes \ell^{2}\left(\mathbb{Z}_{+}\right)$spanned by the vectors $f_{\mu+\left(l-p \wedge \mu_{2}\right) \varepsilon} \otimes e_{p+l-p \wedge \mu_{2}}, l=0, \ldots, N$, with $N=\mu_{1}+p \wedge \mu_{2}$ and $\varepsilon=\varepsilon_{2}-\varepsilon_{1}=(-1,1)$. Note that all these finite-dimensional spaces are orthogonal; $H(\mu, p) \perp H(l, m)$ if $(\mu, p) \neq(l, m)$ and $\ell^{2}\left(\mathbb{Z}_{+}^{2}\right) \otimes \ell^{2}\left(\mathbb{Z}_{+}\right)=$ $\bigoplus_{\mu, p} H(\mu, p)$. It follows from (4.2) and (4.3) that

$$
(\pi \otimes \sigma) T: H(\mu, p) \rightarrow H\left(\mu+\varepsilon_{1}, p\right), \quad(\pi \otimes \sigma) T^{*}: H(\mu, p) \rightarrow H\left(\mu-\varepsilon_{1}, p\right) .
$$

Then $(\pi \otimes \sigma) R: H(\mu, p) \rightarrow H(\mu, p)$ and we show how to diagonalize this finitedimensional mapping. From (4.2) and (4.3) we obtain, with $\varepsilon=\varepsilon_{2}-\varepsilon_{1}$ as before,

$$
\begin{aligned}
((\pi \otimes & \sigma) R)\left(f_{\mu} \otimes e_{k}\right) \\
= & \left(\left(1-q^{2 \mu_{2}}\right)\left(1-q^{2 k}\right)+q^{2+2 k+2 \mu_{2}}\left(1-q^{2 \mu_{1}}\right)\right)\left(f_{\mu} \otimes e_{k}\right) \\
& -q^{k+\mu_{2}+1} \sqrt{\left(1-q^{2 \mu_{1}}\right)\left(1-q^{2 \mu_{2}+2}\right)\left(1-q^{2 k+2}\right)}\left(f_{\mu+\varepsilon} \otimes e_{k+1}\right) \\
& -q^{k+\mu_{2}-1} \sqrt{\left(1-q^{2 \mu_{2}}\right)\left(1-q^{2 \mu_{1}+2}\right)\left(1-q^{2 k}\right)}\left(f_{\mu-\varepsilon} \otimes e_{k-1}\right) .
\end{aligned}
$$

Hence $(\pi \otimes \sigma) R$ on the finite-dimensional space $H(\mu, p)$ is given by a Jacobi matrix, i.e., a tridiagonal symmetric matrix, so the eigenvectors are described in terms of discrete orthonormal polynomials on a finite set. Let us first assume that $p \geq \mu_{2}$. It follows from (4.4) that the vector $\sum_{j=0}^{\mu_{1}+\mu_{2}} p_{j}(\lambda) f_{\mu+\left(j-\mu_{2}\right) \varepsilon} \otimes e_{p+j-\mu_{2}}$ is an eigenvector for $(\pi \otimes \sigma) R$ for the eigenvalue $\lambda$ if and only if

$$
\begin{aligned}
\left(\lambda+q^{2 N}-1\right) p_{j}(\lambda) & =a_{j} p_{j+1}(\lambda)+b_{j} p_{j}(\lambda)+a_{j-1} p_{j-1}(\lambda), \\
\left(\lambda+q^{2 N}-1\right) p_{N}(\lambda) & =b_{N} p_{N}(\lambda)+a_{N-1} p_{N-1}(\lambda),
\end{aligned}
$$


with

$$
\begin{aligned}
& a_{j}=-q^{2 j} \sqrt{q^{2+2 y}\left(1-q^{2 j+2}\right)\left(1-q^{2 N-2 j}\right)\left(1-q^{2 j+2 y+2}\right)}, \\
& b_{j}=q^{2 N}\left(\left(1-q^{2 j-2 N}\right)\left(1-q^{2 y+2+2 j}\right)-q^{2 y+2 j-2 N}\left(1-q^{2 j}\right)\right),
\end{aligned}
$$

where $y=p-\mu_{2}, N=\mu_{1}+\mu_{2}$, and the standard initial conditions $p_{-1}(\lambda)=0$, $p_{0}(\lambda)=1$ hold. Then $p_{j}(\lambda)$ is a polynomial of degree $j$ in $\lambda, 0 \leq j \leq N$.

The polynomial $p_{j}(\lambda)$ can be written in terms of the affine $q$-Krawtchouk polynomial as follows. Recall that the affine $q$-Krawtchouk polynomial is defined by

$$
K_{l}(x)=K_{l}(x ; t, N ; q)={ }_{3} \varphi_{2}\left(\begin{array}{l}
q^{-l}, x, 0 \\
t q, q^{-N}
\end{array} ; q, q\right), \quad 0 \leq l \leq N,
$$

and satisfies the three-term recurrence relation

$$
\begin{aligned}
\left(1-q^{-x}\right) K_{l}\left(q^{-x}\right)= & \left(q^{l-N}-1\right)\left(1-t q^{l+1}\right) K_{l+1}\left(q^{-x}\right) \\
& +\left(\left(1-q^{l-N}\right)\left(1-t q^{n+1}\right)-t q^{l-N}\left(1-q^{l}\right)\right) K_{l}\left(q^{-x}\right) \\
& +t q^{l-N}\left(1-q^{l}\right) K_{l-1}\left(q^{-x}\right),
\end{aligned}
$$

see Stanton [15, §4], Askey and Wilson [2], and references therein. Denote by

$$
\begin{aligned}
\hat{K}_{l}(x) & =\hat{K}_{l}(x ; t, N ; q) \\
& =(-1)^{l}(t q)^{-l / 2}\left(\frac{\left(q^{N} ; q^{-1}\right)_{l}(t q ; q)_{l}}{(q ; q)_{l}}\right)^{1 / 2} K_{l}(x ; t, N ; q)
\end{aligned}
$$

the orthonormal affine $q$-Krawtchouk polynomials with positive leading coefficient, where we assume $0<t q<1$. Then

$$
\begin{aligned}
\left(1-q^{-x}\right) \hat{K}_{l}\left(q^{-x}\right) & =a_{l} \hat{K}_{l+1}\left(q^{-x}\right)+b_{l} \hat{K}_{l}\left(q^{-x}\right)+a_{l-1} \hat{K}_{l-1}\left(q^{-x}\right), \\
\left(1-q^{-x}\right) \hat{K}_{N}\left(q^{-x}\right) & =b_{N} \hat{K}_{N}\left(q^{-x}\right)+a_{N-1} \hat{K}_{N-1}\left(q^{-x}\right),
\end{aligned}
$$

for $x \in\{0, \ldots, N\}$ with

$$
\begin{aligned}
& a_{l}=a_{l}(t, N ; q)=-q^{l-N} \sqrt{t q\left(1-t q^{l+1}\right)\left(1-q^{l+1}\right)\left(1-q^{N-l}\right)}, \\
& b_{l}=b_{l}(t, N ; q)=\left(1-q^{l-N}\right)\left(1-t q^{l+1}\right)-t q^{l-N}\left(1-q^{l}\right) .
\end{aligned}
$$

Comparing both three-term recurrence relations shows that we can make the choice $p_{j}(\lambda)=\hat{K}_{j}\left(q^{-2 x} ; q^{2 y}, N ; q^{2}\right)$ with $\lambda=1-q^{2(N-x)}$ for $x \in \mathbb{Z}_{+}, 0 \leq x \leq N$, and $y=p-\mu_{2}, N=\mu_{1}+\mu_{2}$, as before.

In case $p<\mu_{2}$ we look for an eigenvector of the form $\sum_{j=0}^{p+\mu_{1}} p_{j}(\lambda) f_{\mu+(j-p) \varepsilon} \otimes e_{j}$ and we obtain the same recurrence relation for the polynomials $p_{j}(\lambda)$ as above, but with $y=\mu_{2}-p$ and $N=\mu_{1}+p$. This follows from the symmetry of (4.4) in $k$ and $\mu_{2}$.

Remark 4.1. We have $K_{j}\left(q^{-x} ; t, N ; q\right)=K_{x}\left(q^{-j} ; t, N ; q\right), 0 \leq j, x \leq N$, which means that the affine $q$-Krawtchouk polynomials are self-dual. Using the self-duality and the fact that they form eigenvectors of the self-adjoint operator $(\pi \otimes \sigma) R$ (with other parameters) for different eigenvalues gives the known orthogonality relations [2], [15]

$$
\sum_{j=0}^{N} \frac{\left(q^{N} ; q^{-1}\right)_{j}(t q ; q)_{j}}{(q ; q)_{j}}(t q)^{-j} K_{x}\left(q^{-j}\right) K_{y}\left(q^{-j}\right)=\delta_{x y} \frac{(q ; q)_{x}}{\left(q^{N} ; q^{-1}\right)_{x}(t q ; q)_{x}}(t q)^{x-N}
$$


for the affine $q$-Krawtchouk polynomials $K_{x}\left(q^{-j}\right)=K_{j}\left(q^{-x}\right)=K_{j}\left(q^{-x} ; t, N ; q\right)$. This follows from the previous discussion apart for the occurrence of $(t q)^{-N}$ on the right-hand side of (4.9). It can be evaluated by taking $x=y=0$ and reversing the summation parameter. The resulting sum can be calculated using the $q$-Chu-Vandermonde sum. The corresponding orthogonality measure is positive-definite if $0<t q<1$.

Proposition 4.2. For the $\left(N+1=\mu_{1}+p \wedge \mu_{2}+1\right)$-dimensional subspace $H(\mu, p)$, $\mu \in \mathbb{Z}_{+}^{2}, p \in \mathbb{Z}_{+}$, of the representation space $\ell^{2}\left(\mathbb{Z}_{+}^{2}\right) \otimes \ell^{2}\left(\mathbb{Z}_{+}\right)$corresponding to the representation $\pi \otimes \sigma$ of $\widetilde{\mathcal{X}} \otimes \tilde{\mathcal{Y}}$ there exists an orthogonal basis $\left\{g_{x} \mid x \in\{0, \ldots, N\}\right\}$ of eigenvectors for the positive operator $(\pi \otimes \sigma) R$ (with $R=T T^{*} \in \tilde{\mathcal{X}} \otimes \widetilde{\mathcal{Y}}$ defined by (4.1)). Explicitly, $((\pi \otimes \sigma) R) g_{x}=\left(1-q^{2 x}\right) g_{x}$ and

$$
g_{x}=g_{x}(\mu, p)=\sum_{j=0}^{N} \hat{K}_{j}\left(q^{2 x-2 N} ; q^{2 y}, N ; q^{2}\right) f_{\mu+\left(j-p \wedge \mu_{2}\right) \varepsilon} \otimes e_{p+j-p \wedge \mu_{2}}
$$

with $y=\left|p-\mu_{2}\right|$ and $\varepsilon=\varepsilon_{2}-\varepsilon_{1}$. The orthogonality relations are

$$
\left\langle g_{x}, g_{x^{\prime}}\right\rangle=\delta_{x x^{\prime}} \frac{\left(q^{2} ; q^{2}\right)_{N-x} q^{-2 x(y+1)}}{\left(q^{2 N} ; q^{-2}\right)_{N-x}\left(q^{2 y+2} ; q^{2}\right)_{N-x}} .
$$

Remark 4.3. Instead of taking the representation $\pi$ of $\tilde{\mathcal{X}}$ we can take the representation $\pi_{1}^{\phi}$ of $\widetilde{\mathcal{X}}$ described in Proposition 3.3. Then we can find in a similar way eigenvectors of $\left(\pi_{1}^{\phi} \otimes \sigma\right) R$ in terms of Wall polynomials. Define the orthonormal Wall polynomials by

$$
w_{l}(x ; t ; q)=(-1)^{l}(t q)^{-l / 2}\left(\frac{(t q ; q)_{l}}{(q ; q)_{l}}\right)^{1 / 2}{ }_{2} \varphi_{1}\left(\begin{array}{c}
q^{-l}, 0 \\
t q
\end{array} ; q, q x\right) ;
$$

see, e.g., [10], [17], and references therein. Note that the Wall polynomials are little $q$-Jacobi polynomials (2.3) with $b=0$. The eigenvectors $g_{x}$ of $\left(\pi_{\phi}^{1} \otimes \sigma\right) R$ for the eigenvalue $1-q^{2 x}, x \in \mathbb{Z}_{+}$, in $\ell^{2}\left(\mathbb{Z}_{+}\right) \otimes \ell^{2}\left(\mathbb{Z}_{+}\right)$are

$$
\begin{aligned}
& g_{x}=\sum_{j=0}^{\infty} e^{-i j \phi} w_{j}\left(q^{2 x} ; q^{2 p} ; q^{2}\right) e_{j} \otimes e_{p+j}, \\
& g_{x}=\sum_{j=0}^{\infty} e^{-i j \phi} w_{j}\left(q^{2 x} ; q^{2 p} ; q^{2}\right) e_{p+j} \otimes e_{j},
\end{aligned}
$$

for $p \in \mathbb{Z}_{+}$. For $\phi=0$ these eigenvectors can be obtained by a formal limiting process of the result of Proposition 4.2. Take $\mu_{2}=0$ and let $\mu_{1} \rightarrow \infty$ and use the limit transition

$$
\lim _{N \rightarrow \infty} \hat{K}_{l}\left(x q^{-N} ; t, N ; q\right)=w_{l}(x ; t ; q)
$$

of the affine $q$-Krawtchouk polynomials to the Wall polynomials. So here we let $e_{k} \in$ $\ell^{2}\left(\mathbb{Z}_{+}\right)$correspond to $\lim _{\mu_{1} \rightarrow \infty} f_{\mu_{1}, k}$ in $\ell^{2}\left(\mathbb{Z}_{+}^{2}\right)$. It follows from Proposition 3.3 that under this limit the $*$-representation $\pi$ formally goes over into $\pi_{1}^{0}$.

The occurrence of the Wall polynomials in relation to the eigenvectors of $\left(\pi_{0}^{1} \otimes \sigma\right) R$ as in (4.11) is contained in Koornwinder [10]. To see this, recall, see proof of Proposition 3.3, that $\pi_{0}^{1}$ is a representation of $\widetilde{\mathcal{X}} /\left\langle X_{1}^{*} X_{1}-X_{1}^{*} X_{1}\right\rangle \cong \widetilde{\mathcal{Y}}$, and under this isomorphism $\pi_{0}^{1}=\sigma$. Now $\widetilde{\mathcal{Y}}$ is isomorphic to the $*$-algebra $\mathcal{A}$ of [10, §3] by $Y_{1} \mapsto \gamma, Y_{2} \mapsto \alpha^{*}$, and 
$\pi_{0}^{1}=\sigma$ then corresponds to the representation $\tau$ of [10, (3.6)]. Under this identification $T$ corresponds to $\Phi\left(\alpha^{*}\right)$ of $[10, \S 3]$, where $\Phi$ is the notation for the comultiplication in [10]. Hence $1-R$ corresponds to $1-\Phi\left(\alpha^{*}\right) \phi(\alpha)=\Phi\left(1-\alpha^{*} \alpha\right)=\Phi\left(\gamma \gamma^{*}\right)$, and now $[10,(4.6)]$ corresponds precisely to the vectors in (4.11) being eigenvectors of $\left(\pi_{\phi}^{1} \otimes \sigma\right) R$ for $\phi=0$.

The orthogonality relations for $g_{x}$, i.e., the orthogonality relations dual to the orthogonality relations for the Wall polynomials, now correspond to the orthogonality relations for the Al-Salam-Carlitz polynomials $V_{n}^{(a)}$. Explicitly,

$$
\begin{aligned}
& w_{l}\left(q^{n} ; t ; q\right)=\frac{t^{l / 2} q^{l^{2} / 2}}{\sqrt{(t q, q ; q)_{l}}} 2 \varphi_{0}\left(\begin{array}{c}
q^{-l}, q^{-n} \\
-
\end{array} ; q, \frac{q^{n}}{t}\right) \\
& =\frac{(-t)^{-n} q^{n(n-1) / 2} t^{l / 2} q^{l^{2} / 2}}{\sqrt{(t q, q ; q)_{l}}} V_{n}^{(t)}\left(q^{-l} ; q\right),
\end{aligned}
$$

where the first equality follows from [5, (III.6), (III.7)]. This is implicitly contained in $[10, \S 2]$, but the connection with Al-Salam-Carlitz polynomials is not noticed.

To investigate how the mappings $(\pi \otimes \sigma) T$ and $(\pi \otimes \sigma) T^{*}$ act on the eigenvectors described in Proposition 4.2, we first prove a lemma.

Lemma 4.4. In $\widetilde{\mathcal{X}} \otimes \widetilde{\mathcal{Y}}$ we have the commutation relations

$$
(1-R) T=q^{2} T(1-R), \quad T^{*}(1-R)=q^{2}(1-R) T^{*} .
$$

Proof. Since $R=T T^{*}$ is self-adjoint, it suffices to prove one of the commutation relations. The relation $(1-R) T=q^{2} T(1-R)$ is implied by $T^{*} T-q^{2} T T^{*}=1-q_{\tilde{\tilde{x}}}^{2}$, which is proved directly from (4.1) and the commutation relations (2.1) and (2.2) for $\tilde{\mathcal{X}}$ and $\widetilde{\mathcal{Y}}$.

Remark 4.5. As stated in Section 2 the algebras $\tilde{\mathcal{X}}$ and $\tilde{\mathcal{Y}}$ are closely related to a deformation of the $*$-algebra of polynomials on the sphere in $\mathbb{C}^{n}$ for $n=3$ and $n=2$. This deformation is denoted by $\widetilde{Z}_{n}$ in [4] on which Pol $\left(U_{q}(n)\right)$ acts in a natural way. It is possible to show that $(1-R) T=q^{2} T(1-R)$ follows from the commutation relation $Q_{n-1} z_{n}=q^{2} z_{n} Q_{n-1}$ in $\widetilde{\mathcal{Z}}_{n}$ in the notation of [4] under a suitable algebra homomorphism. This leads to a more conceptual proof of Lemma 4.4, but falls outside the scope of this paper.

Lemma 4.4 implies that $((\pi \otimes \sigma) T) g_{x}(\mu, p)$ is an eigenvector of $(\pi \otimes \sigma)(1-R)$ for the eigenvalue $q^{2 x+2}$ in the space $H\left(\mu+\varepsilon_{1}, p\right)$. Since the eigenvectors $g_{x}\left(\mu+\varepsilon_{1}, p\right)$, $x=0, \ldots, \mu_{1}+p \wedge \mu_{2}+1$, form an orthogonal basis of $H\left(\mu+\varepsilon_{1}, p\right)$, we have proved part of the following proposition.

Proposition 4.6. For $\mu \in \mathbb{Z}_{+}^{2}, p \in \mathbb{Z}_{+}$we have

$$
\begin{gathered}
((\pi \otimes \sigma) T) g_{x}(\mu, p)=-q^{1+y} \sqrt{1-q^{2 N+2}} g_{x+1}\left(\mu+\varepsilon_{1}, p\right), \\
\left((\pi \otimes \sigma) T^{*}\right) g_{x}(\mu, p)=\frac{-q^{-y-1}}{\sqrt{1-q^{2 N}}}\left(1-q^{2 x}\right) g_{x-1}\left(\mu-\varepsilon_{1}, p\right),
\end{gathered}
$$


where $T, T^{*} \in \tilde{\mathcal{X}} \otimes \tilde{\mathcal{Y}}$ are defined by (4.1), $y=\left|p-\mu_{2}\right|, N=\mu_{1}+p \wedge \mu_{2}$, and the vectors $g_{x}(\mu, p) \in \ell^{2}\left(\mathbb{Z}_{+}^{2}\right) \otimes \ell^{2}\left(\mathbb{Z}_{+}\right)$are defined in Proposition 4.2.

Proof. We have that $((\pi \otimes \sigma) T) g_{x}(\mu, p)=C g_{x+1}\left(\mu+\varepsilon_{1}, p\right)$ for some constant $C$, as already remarked. To calculate $C$, take the inner product with $f_{\left(N+1, \mu_{2}-p \wedge \mu_{2}\right)} \otimes e_{p-p \wedge \mu_{2}} \in$ $H\left(\mu+\varepsilon_{1}, p\right)$ and use the fact that we are dealing with a $*$-representation to get

$$
\begin{aligned}
C & =\left\langle C g_{x+1}\left(\mu+\varepsilon_{1}, p\right), f_{\left(N+1, \mu_{2}-p \wedge \mu_{2}\right)} \otimes e_{p-p \wedge \mu_{2}}\right\rangle \\
& =\left\langle g_{x}(\mu, p),\left((\pi \otimes \sigma) T^{*}\right) f_{\left(N+1, \mu_{2}-p \wedge \mu_{2}\right)} \otimes e_{p-p \wedge \mu_{2}}\right\rangle \\
& =-q^{1+y} \sqrt{1-q^{2 N+2}},
\end{aligned}
$$

where we use (4.3) to obtain the last equality. We also have for some other constant $C$ that $\left((\pi \otimes \sigma) T^{*}\right) g_{x}(\mu, p)=C g_{x-1}\left(\mu-\varepsilon_{1}, p\right)$. Now use this and the first statement of the proposition to get

$$
q^{2 x} g_{x}(\mu, p)=\left((\pi \otimes \sigma)\left(1-T T^{*}\right)\right) g_{x}(\mu, p)=\left(1+C q^{1+y} \sqrt{1-q^{2 N}}\right) g_{x}(\mu, p)
$$

from which $C$ follows.

Remark 4.7. From Proposition 4.6 recurrence formulas for affine $q$-Krawtchouk polynomials with shifted parameters can be obtained. Combining Proposition 4.6 with (4.2) and replacing $q^{2}, x-N, q^{2 y}$ by $q,-x, t$, we get

$$
\begin{aligned}
\left(1-q^{N+1}\right) K_{l}\left(q^{-x} ; t, N+1 ; q\right)= & q^{l}\left(1-q^{N-l+1}\right) K_{l}\left(q^{-x} ; t, N ; q\right) \\
& +\left(1-q^{l}\right) K_{l-1}\left(q^{-x} ; t, N ; q\right) .
\end{aligned}
$$

Similarly, combination of Proposition 4.6 with (4.3) gives

$$
\begin{aligned}
\left(1-q^{N-x}\right) K_{l}\left(q^{-x} ; t, N-1 ; q\right)= & t q^{l+1}\left(1-q^{N}\right) K_{l}\left(q^{-x} ; t, N ; q\right) \\
& +\left(1-q^{N}\right)\left(1-t q^{l+1}\right) K_{l+1}\left(q^{-x} ; t, N ; q\right) .
\end{aligned}
$$

These relations are contiguous relations for the ${ }_{3} \varphi_{2}$-series in (4.5).

The next corollary shows how the adjoint of the left-hand side of the addition formula (2.5) under $\pi \otimes \sigma$ acts on the eigenvectors of Proposition 4.2. In order to formulate the corollary we introduce the following notation

$$
r_{l, m}^{(v)}(x ; q)= \begin{cases}(x q ; q)_{l-m}^{1 / 2} p_{m}\left(x ; q^{v}, q^{l-m} ; q\right) & (l \geq m), \\ \left(x ; q^{-1}\right)_{m-l}^{1 / 2} p_{l}\left(x q^{l-m} ; q^{v}, q^{m-l} ; q\right) & (l \leq m),\end{cases}
$$

in terms of the little $q$-Jacobi polynomial (2.3). Note that $r_{l, m}^{(\nu)}\left(x q^{m-l} ; q\right)=r_{m, l}^{(v)}(x ; q)$.

Corollary 4.8. With the notation as in (2.4), (4.1), Proposition 4.2, and with $N=$ $\mu_{1}+p \wedge \mu_{2}, y=\left|p-\mu_{2}\right|$, we have

$$
\begin{aligned}
((\pi \otimes & \left.\sigma) R_{l, m}^{(v)}\left(T, T^{*}, 1 ; q^{2}\right)\right)^{*} g_{x}(\mu, p) \\
= & \left(-q^{y+1}\right)^{m-l}\left(q^{2 N+2} ; q^{2}\right)_{m-l}^{1 / 2}\left(q^{2 x-2 l+2 m+2} ; q^{2}\right)_{l-m}^{1 / 2} \\
& \quad \times r_{l, m}^{(v)}\left(q^{2 x-2 l+2 m} ; q^{2}\right) g_{x+m-l}\left(\mu+(m-l) \varepsilon_{1}, p\right) .
\end{aligned}
$$

Here we use the standard notation $[5](a ; q)_{n}=(a ; q)_{\infty} /\left(a q^{n} ; q\right)_{\infty}$ for $n \in \mathbb{Z}$. 


\section{Addition Formula in Commuting Variables}

In the previous section we have studied the left-hand side of the addition formula (2.5) under the $*$-representation $\pi \otimes \sigma$ of $\widetilde{\mathcal{X}} \otimes \widetilde{\mathcal{Y}}$. In this section, we first study the right-hand side of the addition formula (2.5) under the $*$-representation $\pi \otimes \sigma$ of $\widetilde{\mathcal{X}} \otimes \widetilde{\mathcal{Y}}$. This then directly leads to an addition formula for the functions $r_{l, m}^{(\nu)}(\cdot ; q)$ as defined in (4.13).

First, from (2.4) and Proposition 3.1 we see that the action of $\sigma$ on the second leg of the tensor product of the right-hand side of the addition formula (2.5) is given by

$$
\sigma\left(R_{l-r, m-s}^{(v+r+s)}\left(Y_{2}, Y_{2}^{*}, 1 ; q^{2}\right) Y_{1}^{s}\left(Y_{1}^{*}\right)^{r}\right) e_{k}=q^{k(r+s)} r_{l-r, m-s}^{(v+r+s)}\left(q^{2 k} ; q^{2}\right) e_{k+l-m+s-r} .
$$

Next we calculate the action of $\pi$ on the first leg of the tensor product of the right-hand side of the addition formula (2.5). By iteration of Proposition 3.3 we see that

$$
\begin{aligned}
\pi\left(X_{1}^{p}\right) f_{\mu} & =q^{p \mu_{2}}\left(q^{2 \mu_{1}+2} ; q^{2}\right)_{p}^{1 / 2} f_{\mu+p \varepsilon_{1}}, \\
\pi\left(X_{2}^{p}\right) f_{\mu} & =\left(q^{2 \mu_{2}+2} ; q^{2}\right)_{p}^{1 / 2} f_{\mu+p \varepsilon_{2}}, \\
\pi\left(\left(X_{1}^{*}\right)^{p}\right) f_{\mu} & =q^{p \mu_{2}}\left(q^{2 \mu_{1}} ; q^{-2}\right)_{p}^{1 / 2} f_{\mu-p \varepsilon_{1}}, \\
\pi\left(\left(X_{2}^{*}\right)^{p}\right) f_{\mu} & =\left(q^{2 \mu_{2}} ; q^{-2}\right)_{p}^{1 / 2} f_{\mu-p \varepsilon_{2} .}
\end{aligned}
$$

Consequently, using $\pi\left(1-X_{2} X_{2}^{*}\right) f_{\mu}=q^{2 \mu_{2}} f_{\mu}$ we get

$$
\pi\left(R_{r, s}^{(\nu-1)}\left(X_{1}, X_{1}^{*}, 1-X_{2} X_{2}^{*} ; q^{2}\right)\right) f_{\mu}=q^{\mu_{2}(r+s)} r_{r, s}^{(\nu-1)}\left(q^{2 \mu_{1}} ; q^{2}\right) f_{\mu+(r-s) \varepsilon_{1}}
$$

and

$$
\pi\left(R_{l-r, m-s}^{(\nu+r+s)}\left(X_{2}, X_{2}^{*}, 1 ; q^{2}\right)\right) f_{\mu+(r-s) \varepsilon_{1}}=r_{l-r, m-s}^{(\nu+r+s)}\left(q^{2 \mu_{2}} ; q^{2}\right) f_{\mu+(l-m) \varepsilon_{2}+(s-r) \varepsilon} .
$$

Combination of (5.2) and (5.3) gives the explicit action of the first leg of the tensor product on the right-hand side of the addition formula under the representation $\pi$ on a basis vector.

We now have all the ingredients to turn the addition formula (2.5) into an addition formula in commuting variables for $r_{l, m}^{(v)}(\cdot ; q)$. Apply $\pi \otimes \sigma$ to (2.5) and let the resulting identity act on $f_{l} \otimes e_{z+p-p \wedge \mu_{2}}\left(z \in \mathbb{Z}_{+}\right)$and take inner products with $g_{x}(\mu, p)$. Then use (5.1), (5.2), (5.3), and $\pi \otimes \sigma$ being a $*$-representation in combination with Corollary 4.8, to find

$$
\begin{aligned}
\left(-q^{y+1}\right)^{m-l} & \left(q^{2 N+2} ; q^{2}\right)_{m-l}^{1 / 2}\left(q^{2 x-2 l+2 m+2} ; q^{2}\right)_{l-m}^{1 / 2} r_{l, m}^{(v)}\left(q^{2 x-2 l+2 m} ; q^{2}\right) \\
& \times\left\langle f_{l} \otimes e_{z+p-p \wedge \mu_{2}}, g_{x+m-l}\left(\mu+(m-l) \varepsilon_{1}, p\right)\right\rangle \\
= & \sum_{r=0}^{l} \sum_{s=0}^{m} c_{l, m ; r, s}^{(\nu)}\left(q^{2}\right)(-q)^{r-s} q^{\left(z+p-p \wedge \mu_{2}+\lambda_{2}\right)(r+s)} \\
& \times r_{l-r, m-s}^{(\nu+r+s)}\left(q^{2 \lambda_{2}} ; q^{2}\right) r_{r, s}^{(\nu-1)}\left(q^{2 \lambda_{1}} ; q^{2}\right) r_{l-r, m-s}^{(\nu+r+s)}\left(q^{2\left(z+p-p \wedge \mu_{2}\right)} ; q^{2}\right) \\
& \times\left\langle f_{\lambda+(r-s) \varepsilon_{1}+(l-m+s-r) \varepsilon_{2}} \otimes e_{z+p-p \wedge \mu_{2}+l-m+s-r}, g_{x}(\mu, p)\right\rangle
\end{aligned}
$$

with $y=\left|p-\mu_{2}\right|, N=\mu_{1}+p \wedge \mu_{2}$. The inner products on both sides of (5.4) can be evaluated by use of Proposition 4.2. The left-hand side is nonzero if and only if 
$\lambda_{2}=z+\mu_{2}-p \wedge \mu_{2}, \mu_{1}+\mu_{2}+m-l=\lambda_{1}+\lambda_{2}$ for some $z \in \mathbb{Z}_{+}$with $0 \leq z \leq N+m-l$ and, if so, we find

$$
\left\langle f_{l} \otimes e_{z+p-p \wedge \mu_{2}}, g_{x+m-l}\left(\mu+(m-l) \varepsilon_{1}, p\right)\right\rangle=\hat{K}_{z}\left(q^{2 x-2 N} ; q^{2 y}, N+m-l ; q^{2}\right) .
$$

The inner product on the right-hand side of (5.4) is nonzero if and only if $\lambda_{2}=z+\mu_{2}-$ $p \wedge \mu_{2}, \mu_{1}+\mu_{2}+m-l=\lambda_{1}+\lambda_{2}$ for some $z \in \mathbb{Z}_{+}$with $0 \leq z+l-m+s-r \leq N$ and if so we obtain

$$
\begin{aligned}
& \left\langle f_{\lambda+(r-s) \varepsilon_{1}+(l-m+s-r) \varepsilon_{2}} \otimes e_{z+p-p \wedge \mu_{2}+l-m+s-r}, g_{x}(\mu, p)\right\rangle \\
& \quad=\hat{K}_{z+l-m+s-r}\left(q^{2 x-2 N} ; q^{2 y}, N ; q^{2}\right) .
\end{aligned}
$$

If we interpret $\hat{K}_{z}(x ; t, N ; q)=0$ for $z<0$ and $z>N$, the inner products in (5.5) and (5.6) are well defined for all choices of $z \in \mathbb{Z}$. We now choose $z, N$ such that the conditions $0 \leq z \leq N+m-l$ and $0 \leq z+l-m+s-r \leq N$ are met for all possible choices of $r$ and $s$, which is the case if we take $z+l \leq N$.

Plug (5.5) and (5.6), with the choices $\lambda_{2}=z+\mu_{2}-p \wedge \mu_{2}$ and $\mu_{1}+\mu_{2}+m-l=\lambda_{1}+\lambda_{2}$, in (5.4) to obtain an addition formula in commuting variables. The dependence of the result on the variables $p, \mu_{1}, \mu_{2}$ is only on $N=\mu_{1}+p \wedge \mu_{2}$ and $y=\left|p-\mu_{2}\right|$. Observe that both sides are polynomial in $q^{2 x}$ of degree $z+l$ which is at most equal to $N$, and that it holds for every $x \in\{0, \ldots, N\}$, so that it still holds if we replace $q^{2 x}$ by $x \in \mathbb{C}$. Next observe that if we divide both sides by $q^{y(m-l-z)} \sqrt{\left(q^{2 y+2} ; q^{2}\right)}$ we get an identity which is rational in $q^{2 y}$ and holds for $y \in \mathbb{Z}_{+}$. Hence we can replace $q^{2 y}$ on both sides by $t$ with $0<t<q^{-2}$. Finally, replacing the base $q^{2}$ by $q$ we obtain the addition formula in commuting variables.

Theorem 5.1. The functions $r_{l, m}^{(v)}(x ; q), l, m \in \mathbb{Z}_{+}, v>0$, defined by (4.13), satisfy the addition formula for $x \in \mathbb{C}, t \in\left(0, q^{-1}\right), z, N \in \mathbb{Z}_{+}$with $z+l \leq N$,

$$
\begin{aligned}
& (-1)^{l-m} \sqrt{(t q)^{m-l} \frac{\left(x q^{m-l+1} ; q\right)_{l-m}}{\left(q^{N+m-l+1} ; q\right)_{l-m}}} r_{l, m}^{(v)}\left(x q^{m-l} ; q\right) \hat{K}_{z}\left(x q^{-N} ; t, N+m-l ; q\right) \\
& =\sum_{r=0}^{l} \sum_{s=0}^{m} c_{l, m ; r, s}^{(\nu)}(q)(-1)^{r-s} q^{(1 / 2)(r-s)} t^{(1 / 2)(r+s)} q^{z(r+s)} r_{l-r, m-s}^{(\nu+r+s)}\left(q^{z} ; q\right) r_{l-r, m-s}^{(v+r+s)}\left(t q^{z} ; q\right) \\
& \quad \times r_{r, s}^{(\nu-1)}\left(q^{N+m-l-z} ; q\right) \hat{K}_{z+l-m+s-r}\left(x q^{-N} ; t, N ; q\right)
\end{aligned}
$$

with the notation as in (4.6) and (4.5) and the constants $c_{l, m ; r, s}^{(v)}(q)$ given by (2.6).

Remark 5.2. If we drop the constraint $z+l \leq N$ the addition theorem remains valid for $x$ replaced by $q^{x}(x=0,1, \ldots, N)$.

Remark 5.3. (i) The same result is obtained if we work with the representation $\pi \underset{\sim}{\otimes}$ $\sigma_{\phi}$ of $\widetilde{\mathcal{X}} \otimes \tilde{\mathcal{Y}}$ for arbitrary $\phi \in[0,2 \pi)$. Since $\pi$ is a faithful $*$-representation of $\widetilde{\mathcal{X}}$ and $\bigcap_{\phi} \operatorname{ker}\left(\sigma_{\phi}\right)$ is trivial, we see that the addition formula in commuting variables is equivalent to $(2.5)$. 
(ii) If we work with the representation $\pi_{1}^{\phi} \otimes \sigma$ we obtain the limit case $N \rightarrow \infty$ of Theorem 5.1 (see Remark 4.3). Explicitly, we get

$$
\begin{aligned}
& (-1)^{l-m} \sqrt{(t q)^{m-l}\left(x q^{m-l+1} ; q\right)_{l-m}} r_{l, m}^{(\nu)}\left(x q^{m-l} ; q\right) w_{z}\left(x q^{m-l} ; t ; q\right) \\
& =\sum_{r=0}^{l} \sum_{s=0}^{m} c_{l, m ; r, s}^{(\nu)}(q)(-1)^{r-s} q^{(1 / 2)(r-s)} t^{(1 / 2)(r+s)} q^{z(r+s)} r_{l-r, m-s}^{(\nu+r+s)}\left(q^{z} ; q\right) \\
& \quad \times r_{l-r, m-s}^{(\nu+r+s)}\left(t q^{z} ; q\right) w_{z+l-m+s-r}(x ; t ; q)
\end{aligned}
$$

with the Wall polynomials defined by (4.10).

Remark 5.4. Taking $l=m$ in Theorem 5.1 gives an addition formula for little $q$-Jacobi polynomials $p_{l}\left(\cdot ; q^{v}, 1 ; q\right)$. In order to give it a similar form as the addition formula for the Jacobi polynomial $P_{l}^{(v, 0)}$, see, e.g., [19, Vol. 2, §11.4.2], we split the double sum into two double sums

$$
\begin{aligned}
p_{l}^{(v, 0)} & (x) \hat{K}_{z}\left(x q^{-N} ; t, N ; q\right) \\
= & \sum_{r=0}^{l} \sum_{s=0}^{r}\left(1-\delta_{r s}\right) c_{l, l ; r, s}^{(v)}(q)(-1)^{r-s} q^{(1 / 2)(r-s)} t^{(1 / 2)(r+s)} q^{z(r+s)} \\
& \times\left(q^{z}, t q^{z} ; q^{-1}\right)_{r-s}^{1 / 2}\left(q^{N-z+1} ; q\right)_{r-s}^{1 / 2} \\
& \times p_{l-r}^{(\nu+r+s, r-s)}\left(q^{z+s-r}\right) p_{l-r}^{(\nu+r+s, r-s)}\left(t q^{z+s-r}\right) p_{s}^{(\nu-1, r-s)}\left(q^{N-z}\right) \\
& \times \hat{K}_{z+s-r}\left(x q^{-N} ; t, N ; q\right) \\
& +\sum_{r=0}^{l} \sum_{s=0}^{r} c_{l, l ; r, s}^{(\nu)}(q)(-1)^{r-s} q^{(1 / 2)(r-s)} q^{r^{2}-s^{2}} t^{(1 / 2)(r+s)} q^{z(r+s)} \\
& \times\left(q^{z+1}, t q^{z+1} ; q\right)_{r-s}^{1 / 2}\left(q^{N-z} ; q^{-1}\right)_{r-s}^{1 / 2} \\
& \times p_{l-r}^{(\nu+r+s, r-s)}\left(q^{z}\right) p_{l-r}^{(\nu+r+s, r-s)}\left(t q^{z}\right) p_{s}^{(\nu-1, r-s)}\left(q^{N-z+s-r}\right) \hat{K}_{z+r-s}\left(x q^{-N} ; t, N ; q\right),
\end{aligned}
$$

where we use the notation $p_{n}^{(\alpha, \beta)}(x)=p_{n}\left(x ; q^{\alpha}, q^{\beta} ; q\right)$. Koornwinder's [10] addition formula for the little $q$-Legendre polynomials can be obtained from this identity by first letting $N \rightarrow \infty$ and using the limit transition (4.12) of the affine $q$-Krawtchouk polynomials to the Wall polynomials, or by taking $l=m$ in the degenerate addition formula (5.7). Next let $v \rightarrow 0$. The double sums reduce to single sums, namely, the sums with $s=0$. This is because $\lim _{\nu \rightarrow 0} c_{l, m ; r, s}^{(\nu)}(q)$ is only nonzero if $r=0$ or $s=0$. With this observation Koornwinder's addition formula readily follows.

\section{Product Formula}

In this section, we derive a product formula from the addition formula in Theorem 5.1. For this we introduce a set of orthogonal functions, which allow us to single out the $r=s=0$ term in the addition formula.

For $a \in \mathbb{Z}_{+}$fixed and $s, r, N \in \mathbb{Z}_{+}$such that $0 \leq a \leq N$ we define

$$
P_{r, s}^{(v)}(x, N ; a, t ; q)=r_{r, s}^{(v)}\left(q^{N-a} ; q\right) \hat{K}_{a+s-r}\left(x q^{-N} ; t, N ; q\right),
$$


where we use the notation as in (4.6) and (4.13) and the convention that $\hat{K}_{l}(x ; t, N ; q)=$ 0 whenever $l<0$ or $l>N$. Note that $P_{r, s}^{(v)}(x, N ; a, t ; q)$ is a polynomial in $x$.

Proposition 6.1. For $v>-1$ the functions $P_{r, s}^{(v)}(x, N)=P_{r, s}^{(v)}(x, N ; a, t ; q)$ satisfy the orthogonality relations

$$
\begin{gathered}
\sum_{N=a}^{\infty} \sum_{x=0}^{N} P_{r, s}^{(v)}\left(q^{x}, N\right) P_{r^{\prime}, s^{\prime}}^{(v)}\left(q^{x}, N\right) \frac{\left(q^{N} ; q^{-1}\right)_{N-x}(t q ; q)_{N-x}}{(q ; q)_{N-x}}(t q)^{x} q^{(v+1)(N-a)} \\
=\delta_{r r^{\prime}} \delta_{s s^{\prime}} h_{r s},
\end{gathered}
$$

where

$$
h_{r s}=\frac{(q ; q)_{r}(q ; q)_{s}}{\left(q^{v+1} ; q\right)_{r}\left(q^{v+1} ; q\right)_{s}} \frac{q^{(v+1) s}}{1-q^{v+r+s+1}} .
$$

Proof. This follows from the orthogonality relations (4.9) for the affine $q$-Krawtchouk polynomials, the orthogonality relations for the little $q$-Jacobi polynomials [1], [5]

$$
\sum_{x=0}^{\infty} \frac{(b q ; q)_{x}}{(q ; q)_{x}}(a q)^{x}\left(p_{l} p_{m}\right)\left(q^{x} ; a, b ; q\right)=\delta_{l m} \frac{(q, b q ; q)_{l}(a q)^{l}(1-a b q)\left(a b q^{2} ; q\right)_{\infty}}{(a q, a b q ; q)_{l}\left(1-a b q^{2 l+1}\right)(a q ; q)_{\infty}}
$$

with $0<a q<1, b<q^{-1}$ and the definition (4.13).

The addition formula of Theorem 5.1 can be viewed as the expansion of the lefthand side in terms of the orthogonal functions $P_{r, s}^{(v-1)}(x, N ; z+l-m, t ; q)$. Using the orthogonality relations of Proposition 6.1, we can pick out the term $r=s=0$ to find a product formula.

Theorem 6.2. The functions $r_{l, m}^{(v)}(x ; q), l, m \in \mathbb{Z}_{+}, v>0$, defined by (4.13), satisfy the following product formula for $t \in\left(0, q^{-1}\right), z \in \mathbb{Z}_{+}$:

$$
\begin{aligned}
r_{l, m}^{(\nu)}\left(q^{z} ; q\right) r_{l, m}^{(\nu)}\left(t q^{z} ; q\right)= & \left(1-q^{\nu}\right) \sum_{N=z+l-m}^{\infty} \sum_{x=0}^{N}(-1)^{l-m} \sqrt{(t q)^{m-l} \frac{\left(q^{x-l+m+1} ; q\right)_{l-m}}{\left(q^{N+m-l+1} ; q\right)_{l-m}}} \\
& \times \frac{\left(q^{N} ; q^{-1}\right)_{N-x}(t q ; q)_{N-x}}{(q ; q)_{N-x}}(t q)^{x} q^{\nu(N-z-l+m)} r_{l, m}^{(\nu)}\left(q^{x+m-l} ; q\right) \\
& \times \hat{K}_{z}\left(q^{x-N} ; t, N+m-l ; q\right) \hat{K}_{z+l-m}\left(q^{x-N} ; t, N ; q\right)
\end{aligned}
$$

with the notation as in (4.6).

Notice that the inner summation, in fact, runs from the maximum of 0 and $l-m$ to $N$.

Remark 6.3. The symmetry relation $r_{l, m}^{(v)}\left(x q^{m-l} ; q\right)=r_{m, l}^{(v)}(x ; q)$ gives rise to a symmetry of the product formula (6.2). Namely, changing $x, z, N$ to $x+l-m, z+m-$ $l, N+l-m$ on both sides of (6.2) has the same effect as interchanging the parameters $l$ and $m$. In particular, the full identity (6.2) is known when it is known either for $l \leq m$ or $l \geq m$. 
By specializing $l=m$ we obtain the following corollary for the little $q$-Jacobi polynomials.

Corollary 6.4. The little $q$-Jacobi polynomials $p_{l}\left(x ; q^{v}, 1 ; q\right), l \in \mathbb{Z}_{+}, v>0$, defined by (2.3), satisfy the following product formula for $t \in\left(0, q^{-1}\right), z \in \mathbb{Z}_{+}$:

$$
\begin{aligned}
p_{l}\left(q^{z} ; q^{v}, 1 ; q\right) p_{l}\left(t q^{z} ; q^{v}, 1 ; q\right)= & \left(1-q^{\nu}\right) \sum_{N=z}^{\infty} \sum_{x=0}^{N} \frac{\left(q^{N} ; q^{-1}\right)_{N-x}(t q ; q)_{N-x}}{(q ; q)_{N-x}}(t q)^{x} \\
& \times q^{\nu(N-z)} p_{l}\left(q^{x} ; q^{\nu}, 1 ; q\right)\left(\hat{K}_{z}\left(q^{x-N} ; t, N ; q\right)\right)^{2} .
\end{aligned}
$$

Remark 6.5. One can show, by use of Abel's partial summation formula, that in the limit $v \rightarrow 0$ the right-hand side in Corollary 6.4, at least formally, tends to

$$
\sum_{x=0}^{\infty} \frac{\left(q^{x+1} ; q\right)_{\infty}(t q ; q)_{\infty}}{(q ; q)_{\infty}}(t q)^{x} p_{l}\left(q^{x} ; 1,1 ; q\right)\left(w_{z}\left(q^{x} ; t ; q\right)\right)^{2}
$$

with $w_{z}(\cdot ; t ; q)$ as in (4.10). After some rewriting it is easily seen that for $t=q^{y}$ this is the same expression as the one derived by Koornwinder [10, Theorem 5.1].

\section{The Limit Case $q \uparrow 1$}

In this section we consider the limit case $q \uparrow 1$ of the addition and product formula for the little $q$-disk polynomials to the addition and product formula (1.3) and (1.4) for the disk polynomials. For this limit transition we use the methods developed by Van Assche and Koornwinder [17]. Since some of the calculations are rather tedious we restrict ourselves in this section to giving the key steps; details can be found in the Appendix. Calculations similar to the ones appearing here were made in [8].

First we apply [17, Theorem 1] to the polynomial $\hat{K}_{l}\left(x q^{-N} ; t, N+a ; q\right)$. It gives the limit of the quotient of two such polynomials as $q$ tends to 1. Although [17, Theorem 1] is not formulated for discrete orthogonal polynomials, the proof of [17, Theorem 1] is also applicable to discrete orthogonal polynomials provided that $N$, and hence the number of orthogonal polynomials, tends to infinity. It is straightforward to verify that the conditions of [17, Theorem 1] are satisfied. Explicitly, we have the following result.

Proposition 7.1. For $0<t<c^{-1}, c \in(0,1), a, m \in \mathbb{Z}$, and $N \in \mathbb{N}$, we have

$$
\lim _{n \rightarrow \infty} \frac{\hat{K}_{n+m}\left(x\left(c^{1 / n}\right)^{-n N} ; t, n N+a ; c^{1 / n}\right)}{\hat{K}_{n}\left(x\left(c^{1 / n}\right)^{-n N} ; t, n N+a ; c^{1 / n}\right)}=\rho^{m}((x-B) /(2 A)),
$$

uniformly for $x$ on compact subsets of $\mathbb{C} \backslash\left[0, c^{-a}\right]$. Here

$$
A=c \sqrt{t(1-t c)(1-c)\left(1-c^{N-1}\right)}>0, \quad B=c+t c-2 t c^{2}+t c^{1+N} \in \mathbb{R},
$$

and $\rho(x)=x+\sqrt{x^{2}-1}$ with the square root taken such that $|\rho(x)|>1$ for $x \in$ $\mathbb{C} \backslash[-1,1]$.

In order to apply Proposition 7.1 to the addition formula we need the connection coefficients between affine $q$-Krawtchouk polynomials for different $N$. 
Proposition 7.2. For $a \in \mathbb{Z}, a \leq N, x \in \mathbb{C}$, and $0 \leq l \leq N \wedge(N-a)$, we have the connection formula

$$
K_{l}(x ; t, N ; q)=\sum_{k=0}^{l} \frac{\left(q^{l} ; q^{-1}\right)_{k}}{(q ; q)_{k}} q^{(-N+a) k} \frac{\left(q^{-a} ; q\right)_{k}}{\left(q^{-N} ; q\right)_{l}}\left(q^{-N+a} ; q\right)_{l-k} K_{l-k}(x ; t, N-a ; q) \text {. }
$$

Proof. There are several ways of proving the proposition. We use the generating function for the affine $q$-Krawtchouk polynomials; for $x \in\{0, \ldots, N\}$,

$$
\left(z q^{-N} ; q\right)_{N-x} \varphi_{1}\left(q^{-x} ; t q ; q, t q z\right)=\sum_{l=0}^{N} \frac{\left(q^{-N} ; q\right)_{l}}{(q ; q)_{l}} K_{l}\left(q^{-x} ; t, N ; q\right) z^{l}
$$

This identity is proved as follows: insert (4.5) in the right-hand side of (7.1), change summations, and apply the $q$-binomial theorem to find that this right-hand side equals

$$
\left(z q^{-N} ; q\right)_{N 3} \phi_{2}\left[\begin{array}{c}
q^{-x}, 0,0 \\
t q, q / z
\end{array} ; q, q\right] .
$$

Then use the limiting case $b \rightarrow \infty$ of the identity which one gets by equating [5, (III.5)] and the right-hand side of [5, (III.4)], with $a=q^{-x}, c=t q$ and $z$ replaced by $t q z / b$, to obtain the left-hand side of (7.1). Now, for $|z|<q^{N-a}$

$$
\begin{aligned}
& \sum_{l=0}^{N} \frac{\left(q^{-N} ; q\right)_{l}}{(q ; q)_{l}} K_{l}\left(q^{-x} ; t, N ; q\right) z^{l} \\
& =\left(z q^{-N} ; q\right)_{a}\left(z q^{-N+a} ; q\right)_{N-a-x} \varphi_{1}\left(q^{-x} ; t q ; q, t q z\right) \\
& =\sum_{k=0}^{\infty} \frac{\left(q^{-a} ; q\right)_{k}}{(q ; q)_{k}}\left(z q^{-N+a}\right)^{k} \sum_{j=0}^{N-a} \frac{\left(q^{-N+a} ; q\right)_{j}}{(q ; q)_{j}} K_{j}\left(q^{-x} ; t, N-a ; q\right) z^{j} \\
& =\sum_{l=0}^{N}\left(\sum_{k=0}^{l} \frac{\left(q^{-a} ; q\right)_{k}}{(q ; q)_{k}} q^{(-N+a) k} \frac{\left(q^{-N+a} ; q\right)_{l-k}}{(q ; q)_{l-k}} K_{l-k}\left(q^{-x} ; t, N-a ; q\right)\right) z^{l}
\end{aligned}
$$

by the $q$-binomial theorem and (7.1). Observe that the right-hand side is, in fact, a polynomial because of the convention that $K_{l}(x ; t, N ; q)=0$ whenever $l>N$. This proves the proposition for $x$ replaced by $q^{-x}, x \in\{0, \ldots, N \wedge(N-a)\}$. Since both sides are polynomials of degree not exceeding $N \wedge(N-a)$ the result follows for arbitrary $x \in \mathbb{C}$.

Next use Propositions 7.1 and 7.2 and the binomial theorem to obtain the limit of the quotient of the affine $q$-Krawtchouk polynomials appearing in the addition formula of Theorem 5.1. We replace $q, z, N$ by $c^{1 / n z}, n z, n z N$ for $c \in(0,1)$ and $z, N, n \in \mathbb{N}$, and apply the binomial theorem to find

$$
\lim _{n \rightarrow \infty} \frac{\hat{K}_{n z+l-m+s-r}\left(x\left(c^{1 / n z}\right)^{-n z N} ; t, n z N ; c^{1 / n z}\right)}{\hat{K}_{n z}\left(x\left(c^{1 / n z}\right)^{-n z N} ; t, n z N+m-l ; c^{1 / n z}\right)}
$$




$$
\begin{aligned}
= & \left(c \sqrt{\frac{1-c^{N-1}}{1-c^{N}}}\right)^{l-m} \rho^{l-m+s-r}\left(\frac{x-B}{2 A}\right) \\
& \times\left(\frac{1+\sqrt{(1-t c)(1-c)\left(1-c^{N-1}\right)}}{\sqrt{t} c^{N}\left(1-c^{-N+1}\right) \rho((x-B) / 2 A)}\right)^{l-m}
\end{aligned}
$$

uniformly in $x$ on compact sets of $\mathbb{C} \backslash\left[0, c^{m-l}\right]$. Here $A$ and $B$ are as in Proposition 7.1. We remark that in case $m-l \geq 0$ the sum obtained on the left from applying the connection formula of Proposition 7.2 to the numerator polynomial is finite, even when we let $n$ tend to infinity. If $m-l \leq 0$, one develops the denominator polynomial as a (finite) sum by means of the connection formula.

Introduce the parameter $u>1$ by putting $(x-B) /(2 A)=\frac{1}{2}\left(u+u^{-1}\right)$. Then $\rho((x-$ $B) /(2 A))=u$. If we now divide both sides of the addition formula in Theorem 5.1 by the polynomial $\left.\hat{K}_{z}\left(x q^{-N} ; t, N+m-l ; q\right)\right)$ and, as before, replace $q, z, N$ by $c^{1 / n z}, n z$, $n z N$ and let $n$ tend to infinity, then, after some careful computations using the above result and an analytic continuation argument replacing $-u^{-1}$ by $e^{i \psi}$, we end up with the identity

$$
\begin{aligned}
R_{l, m}^{(v)}(z)= & \sum_{r=0}^{l} \sum_{s=0}^{m} C_{l, m ; r, s}^{(\nu)}\left(\sin \theta_{1}\right)^{r+s} R_{l-r, m-s}^{(v+r+s)}\left(\cos \theta_{1}\right) \\
& \times\left(\sin \theta_{2}\right)^{r+s} R_{l-r, m-s}^{(v+r+s)}\left(\cos \theta_{2}\right) R_{r, s}^{(\nu-1)}\left(\rho e^{i \psi}\right) .
\end{aligned}
$$

Here $\cos \theta_{1}=\sqrt{1-c}, \cos \theta_{2}=\sqrt{1-t c}, \rho=\sqrt{1-c^{N-1}}$, and $z=\sqrt{1-c} \sqrt{1-t c}+$ $c \sqrt{t} \sqrt{1-c^{N-1}} e^{i \psi}=\cos \theta_{1} \cos \theta_{2}+\sin \theta_{1} \sin \theta_{2} \rho e^{i \psi}$. In order to obtain the general classical addition formula for disk polynomials (1.3) from this, one simply observes that multiplication of both sides of (7.2) with $e^{i(l-m) \phi_{1}} e^{i(l-m) \phi_{2}}$ by homogeneity of the polynomials has the effect of replacing $\cos \theta_{1}$ by $e^{i \phi_{1}} \cos \theta_{1}$ and $\cos \theta_{2}$ by $e^{i \phi_{2}} \cos \theta_{2}$, and hence produces the full classical identity.

Let us turn now to the product formula. In view of Remark 6.3 we can restrict ourselves to the case $l \leq m$. Observe that the right-hand side of (6.2) is of the form

$$
\left(1-q^{\nu}\right) \sum_{N=z+l-m}^{\infty} q^{\nu(N-z)} g(N, z, q)
$$

with

$$
\begin{aligned}
g(N, z, q)= & \sum_{x=0}^{N}(-1)^{l-m} \sqrt{(t q)^{m-l} \frac{\left(q^{x-l+m+1} ; q\right)_{l-m}}{\left(q^{N+m-l+1} ; q\right)_{l-m}}} \\
& \times \frac{\left(q^{N} ; q^{-1}\right)_{N-x}(t q ; q)_{N-x}}{(q ; q)_{N-x}}(t q)^{x} r_{l, m}^{(\nu)}\left(q^{x+m-l} ; q\right) \\
& \times \hat{K}_{z}\left(q^{x-N} ; t, N+m-l ; q\right) \hat{K}_{z+l-m}\left(q^{x-N} ; t, N ; q\right) .
\end{aligned}
$$

If we replace $q, z, N$ in (7.3) by $c^{1 / n z}, n z, n z N$, with $c \in(0,1)$, and let $n$ tend to infinity, we formally get

$$
\lim _{n \rightarrow \infty} n z\left(1-c^{v / n z}\right) \sum_{\substack{N=1 \\ \text { step-size } 1 / n z}}^{\infty} c^{v(N-1)} \frac{g\left(n z N, n z, c^{1 / n z}\right)}{n z}
$$




$$
\begin{aligned}
& =-v \log c \int_{N=1}^{\infty} c^{v(N-1)} \lim _{n \rightarrow \infty}\left(\frac{g\left(n z N, n z, c^{1 / n z}\right)}{n z}\right) d N \\
& =-v \log c \int_{N=1}^{\infty} c^{v(N-1)} G\left(\sqrt{1-c^{N-1}}\right) d N \\
& =v \int_{0}^{1} G(\sqrt{1-\rho}) \rho^{v-1} d \rho,
\end{aligned}
$$

where we made the substitution $\rho=c^{N-1}$ in the last step. To calculate the limit function $G$ and to show that it, as a function of $N$, in fact only depends on $\sqrt{1-c^{N-1}}$, we proceed as follows. Use the connection formula of Proposition 7.2 to replace the Krawtchouk polynomial $\hat{K}_{z}\left(q^{x-N} ; t, N+m-l ; q\right)$ in (7.4) by a sum of polynomials $\hat{K}_{z-k}\left(q^{x-N} ; t, N ; q\right)(k=0, \ldots, z)$. Then replace $q, z, N$ by $c^{1 / n z}, n z, n z N$ for $c \in(0,1)$, and send $n$ to infinity. According to [17, Theorem 2], which again is valid also for discrete orthogonal polynomials, and the binomial theorem we get

$$
\begin{aligned}
G(N)= & (-1)^{l-m}\left[c \sqrt{t\left(1-c^{N-1}\right)}\right]^{m-l} \sum_{k=0}^{m-l}(-1)^{k} t^{-k / 2} c^{-N k} \frac{(l-m)_{k}}{k !} \\
& \times\left[(1-c)(1-t c)\left(1-c^{N-1}\right)\right]^{k / 2}\left(1-c^{-N+1}\right)^{-k} \\
& \times \frac{1}{\pi} \int_{B-2 A}^{B+2 A}(1-x)^{(l-m) / 2} R_{l, m}^{(v)}(\sqrt{1-x}) \frac{T_{m-l-k}((x-B) / 2 A)}{\sqrt{4 A^{2}-(x-B)^{2}}} d x,
\end{aligned}
$$

where $T_{n}$ are Chebyshev polynomials of the first kind and $A, B$ are as in Proposition 7.1. Next perform the substitution $(x-B) /(2 A)=\frac{1}{2}\left(u+u^{-1}\right)=\cos \psi$ with $u=e^{i \psi}$. Then interchange the order of summation and integration and use that $T_{n}(\cos \psi)=$ $\frac{1}{2}\left(e^{i n \psi}+e^{-i n \psi}\right)$ to get two finite sums. Apply the binomial theorem to both of these sums to obtain

$$
\begin{aligned}
G(N)= & (-1)^{l-m}\left[c \sqrt{t\left(1-c^{N-1}\right)}\right]^{m-l} \\
& \times \frac{1}{2 \pi} \int_{0}^{\pi}(1-(2 A \cos \psi+B))^{(l-m) / 2} R_{l, m}^{(\nu)}(\sqrt{1-(2 A \cos \psi+B)}) \\
& \times\left\{\left(e^{i \psi}+\sqrt{t^{-1}(1-c)(1-t c)\left(1-c^{N-1}\right)} \frac{c^{-N}}{1-c^{-N+1}}\right)^{m-l}\right. \\
& \left.+\left(e^{-i \psi}+\sqrt{t^{-1}(1-c)(1-t c)\left(1-c^{N-1}\right)} \frac{c^{-N}}{1-c^{-N+1}}\right)^{m-l}\right\} d \psi
\end{aligned}
$$

As we assumed that $l \leq m$, we have

$$
(1-(2 A \cos \psi+B))^{(l-m) / 2} R_{l, m}^{(v)}(\sqrt{1-(2 A \cos \psi+B)})=P_{l}^{(v, l-m)}(1-2(2 A \cos \psi+B))
$$

with $A, B$ as in Proposition 7.1. It is easily checked that the following equality holds:

$$
\begin{aligned}
1-(2 A \cos \psi+B)= & \left(\sqrt{(1-c)(1-t c)}-c \sqrt{t\left(1-c^{N-1}\right)} e^{i \psi}\right) \\
& \times\left(\sqrt{(1-c)(1-t c)}-c \sqrt{t\left(1-c^{N-1}\right)} e^{-i \psi}\right) .
\end{aligned}
$$


When we use this, we find that the right-hand side of (7.6) reduces to

$$
\begin{aligned}
& \frac{1}{2 \pi} \int_{0}^{\pi} P_{l \wedge m}^{(\nu,|l-m|)}(1-2(2 A \cos \psi+B)) \\
& \quad \times\left\{\left(\sqrt{(1-c)(1-t c)}-c \sqrt{t\left(1-c^{N-1}\right)} e^{-i \psi}\right)^{m-l}\right. \\
& \left.\quad+\left(\sqrt{(1-c)(1-t c)}-c \sqrt{t\left(1-c^{N-1}\right)} e^{i \psi}\right)^{m-l}\right\} d \psi \\
& =\frac{1}{2 \pi} \int_{0}^{2 \pi} P_{l \wedge m}^{(\nu,|l-m|)}(1-2(2 A \cos \psi+B)) \\
& \quad \times\left(\sqrt{(1-c)(1-t c)}+c \sqrt{t\left(1-c^{N-1}\right)} e^{-i \psi}\right)^{m-l} d \psi,
\end{aligned}
$$

where in the second step we combined the two integrals and changed $\psi$ to $\psi-\pi$.

So indeed we see that $G$, as a function of $N$, only depends on $\sqrt{1-c^{N-1}}$. Combining this result with (7.5) we find that we end up with

$$
\begin{aligned}
\frac{v}{2 \pi} & \int_{0}^{1} \int_{0}^{2 \pi} P_{l \wedge m}^{(\nu,|l-m|)}(1-2(2 A \cos \psi+B)) \\
& \times\left(\sqrt{(1-c)(1-t c)}+c \sqrt{t} \sqrt{1-\rho} e^{-i \psi}\right)^{m-l} \rho^{\nu-1} d \psi d \rho \\
= & \frac{v}{\pi} \int_{0}^{1} \int_{0}^{2 \pi} P_{l \wedge m}^{(\nu,|l-m|)}\left(2|z|^{2}-1\right)(\bar{z})^{m-l} r\left(1-r^{2}\right)^{\nu-1} d \psi d r \\
= & \frac{v}{\pi} \int_{0}^{1} \int_{0}^{2 \pi} R_{l, m}^{(\nu)}(z) r\left(1-r^{2}\right)^{\nu-1} d \psi d r
\end{aligned}
$$

where we substituted $r=\sqrt{1-\rho}$ and where $z=\sqrt{(1-c)(1-t c)}+c \sqrt{t} r e^{i \psi}$.

It is easily seen from the limit transition of the little $q$-Jacobi polynomials to the Jacobi polynomials that, under the above substitutions, the left-hand side of the product formula (6.2) tends to the product

$$
R_{l, m}^{(v)}(\sqrt{1-c}) R_{l, m}^{(v)}(\sqrt{1-t c})
$$

as $n$ tends to infinity. From the fact that (7.7) equals (7.8) we obtain the full classical product formula (1.4) when we substitute $\cos \theta_{1}$ and $\cos \theta_{2}$ for $\sqrt{1-c}$ and $\sqrt{1-t c}$, respectively, and invoke the same homogeneity argument as in the case of the limit transition of the addition formula. Since the case $l \geq m$ followed from a symmetry argument, we are done.

\section{Appendix}

In this appendix we give justifications for, and details of, the calculations that were made in Section 7.

To prove Proposition 7.1 we have to show that all the conditions of [17, Theorem 1] are satisfied. From the recurrence relation (4.7) we obtain $(a \in \mathbb{Z}, z, N, n \in \mathbb{N}, c \in(0,1))$

$$
x \hat{K}_{n z}\left(x c^{-N} ; t, n z N+a ; c^{1 / n z}\right)
$$




$$
\begin{aligned}
= & -c^{N} a_{n z}\left(t, n z N+a ; c^{1 / n z}\right) \hat{K}_{n z+1}\left(x c^{-N} ; t, n z N+a ; c^{1 / n z}\right) \\
& +c^{N}\left(1-b_{n z}\left(t, n z N+a ; c^{1 / n z}\right)\right) \hat{K}_{n z}\left(x c^{-N} ; t, n z N+a ; c^{1 / n z}\right) \\
& -c^{N} a_{n z-1}\left(t, n z N+a ; c^{1 / n z}\right) \hat{K}_{n z-1}\left(x c^{-N} ; t, n z N+a ; c^{1 / n z}\right)
\end{aligned}
$$

with $a_{l}(t, N ; q)$ and $b_{l}(t, N ; q)$ given by (4.8). Put

$$
\hat{a}_{l}(t, N ; q)=-q^{N} a_{l}(t, N ; q), \quad \hat{b}_{l}(t, N ; q)=q^{N}\left(1-b_{l}(t, N ; q)\right) .
$$

Then it is immediate that

$$
\begin{aligned}
& \lim _{n \rightarrow \infty} \hat{a}_{n z}\left(t, n z N+a ; c^{1 / n z}\right)=A, \\
& \lim _{n \rightarrow \infty} \hat{b}_{n z}\left(t, n z N+a ; c^{1 / n z}\right)=B,
\end{aligned}
$$

with

$$
A=c \sqrt{t(1-t c)(1-c)\left(1-c^{N-1}\right)}>0, \quad B=c+t c-2 t c^{2}+t c^{1+N} \in \mathbb{R} ;
$$

see Proposition 7.1. Furthermore, the expressions $\hat{a}_{l}\left(t, n z N+a ; c^{1 / n z}\right)^{2}$ and $\hat{b}_{l}(t, n z N+$ $\left.a ; c^{1 / n z}\right)$ are both of the form $f\left(c^{1 / n z}, c^{l / n z}\right)$ with $f$ a polynomial. Hence, by Lipschitz continuity, we find that

$$
\begin{aligned}
\lim _{n \rightarrow \infty}\left[\hat{a}_{l}\left(t, n z N+a ; c^{1 / n z}\right)^{2}-\hat{a}_{l-1}\left(t, n z N+a ; c^{1 / n z}\right)^{2}\right] & =0, \\
\lim _{n \rightarrow \infty}\left[\hat{b}_{l}\left(t, n z N+a ; c^{1 / n z}\right)-\hat{b}_{l-1}\left(t, n z N+a ; c^{1 / n z}\right)\right] & =0,
\end{aligned}
$$

uniformly in $l$. The support of the orthogonality measure of the polynomials $\hat{K}_{l}(x ; t, N ; q)$ is contained in the interval $\left[1, q^{-N}\right]$, hence the polynomials

$$
x \mapsto \hat{K}_{l}\left(x c^{-N} ; t, n z N+a ; c^{1 / n z}\right)
$$

have a measure of orthogonality with support in $\left[c^{N}, c^{-a / n z}\right]$. A given compact subset of $\mathbb{C} \backslash[0,1]$ will have no intersection with $\left[c^{N}, c^{-a / n z}\right]$ if $n$ is big enough (this is trivial when $a \leq 0$ ). From the proof of [17, Theorem 1] it follows that the statement of that theorem is still valid for orthogonal polynomials with finite support, provided that the support of the orthogonality measure for the polynomials $p_{l}(\cdot ; n)$ contains more than $n+2$ points, which is true in our case. In this way we have verified that all the conditions of [17, Theorem 1] are fulfilled, which proves Proposition 7.1.

As to the explicit calculations, observe that from Proposition 7.2 we obtain the following connection formula for the orthonormal affine $q$-Krawtchouk polynomials:

(A.1) $\hat{K}_{l}(x ; t, N ; q)=\sum_{k=0}^{l}(-1)^{k}(t q)^{-k / 2} q^{(-N+a) k} \frac{\left(q^{-a} ; q\right)_{k}\left(q^{-N+a} ; q\right)_{l-k}}{(q ; q)_{k}\left(q^{-N} ; q\right)_{l}}$

$$
\begin{aligned}
& \times\left[\left(t q^{l} ; q^{-1}\right)_{k}\left(q^{N} ; q^{-1}\right)_{a}\left(q^{N-l+1} ; q\right)_{k-a}\left(q^{l} ; q^{-1}\right)_{k}\right]^{\frac{1}{2}} \\
& \times \hat{K}_{l-k}(x ; t, N-a ; q) \\
= & \sum_{k=0}^{l}(-1)^{k}(t q)^{-k / 2} q^{(-N+a) k} \\
& \times \frac{\left(q^{-a} ; q\right)_{k}}{(q ; q)_{k}\left(q^{-N} ; q\right)_{a}\left(q^{-N+l-1} ; q^{-1}\right)_{k-a}} \\
& \times\left[\left(t q^{l} ; q^{-1}\right)_{k}\left(q^{N} ; q^{-1}\right)_{a}\left(q^{N-l+1} ; q\right)_{k-a}\left(q^{l} ; q^{-1}\right)_{k}\right]^{1 / 2} \\
& \times \hat{K}_{l-k}(x ; t, N-a ; q)
\end{aligned}
$$


for $a \leq N, 0 \leq l \leq N \wedge(N-a)$. Now consider the quotient

$$
\frac{\hat{K}_{z+l-m+s-r}\left(x q^{-N} ; t, N ; q\right)}{\hat{K}_{z}\left(x q^{-N} ; t, N+m-l ; q\right)} .
$$

Suppose $a=l-m \geq 0$. Then the sum in (A.1) will be a finite sum $k=0, \ldots, a$ because of the factor $\left(q^{-a} ; q\right)_{k}$. So if we develop the numerator of (A.2) in terms of polynomials $\hat{K}_{z+l-m+s-r-k}\left(x q^{-N} ; t, N ; q\right)(k=0, \ldots, z+l-m+s-r)$ the sum will always be finite in this case. Replacing $q, z, N$ by $c^{1 / n z}, n z, n z N$ with $c \in(0,1)$ and $n, z, N \in \mathbb{N}$, and applying Proposition 7.1 we then obtain

$$
\begin{aligned}
\lim _{n \rightarrow \infty} & \frac{\hat{K}_{n z+l-m+s-r}\left(x\left(c^{1 / n z}\right)^{-n z N} ; t, n z N ; c^{1 / n z}\right)}{\hat{K}_{n z}\left(x\left(c^{1 / n z}\right)^{-n z N} ; t, n z N+m-l ; c^{1 / n z}\right)} \\
= & \sum_{k=0}^{l-m}(-1)^{k} t^{-1 / 2 k} c^{-N k} \frac{(m-l)_{k}}{k !}\left(1-c^{-N}\right)^{m-l}\left(1-c^{-N+1}\right)^{l-m-k} \\
& \times\left[(1-t c)^{k}\left(1-c^{N}\right)^{l-m}\left(1-c^{N-1}\right)^{k-l+m}(1-c)^{k}\right]^{1 / 2} \\
& \times \rho^{l-m+s-r-k}\left(\frac{x-B}{2 A}\right) \\
= & \left.\frac{1-c^{-N+1}}{1-c^{-N}}\right]^{l-m}\left[\frac{1-c^{N}}{1-c^{N-1}}\right]^{(1 / 2)(l-m)} \rho^{l-m+s-r}\left(\frac{x-B}{2 A}\right) \\
& \times \sum_{k=0}^{l-m} \frac{(m-l)_{k}}{k !}\left[-\frac{\sqrt{t^{-1}(1-c)(1-t c)\left(1-c^{N-1}\right)}}{c^{N}\left(1-c^{-N+1}\right) \rho((x-B) / 2 A)}\right]^{k} \\
= & c^{l-m}\left[\frac{1-c^{N-1}}{1-c^{N}}\right]^{(1 / 2)(l-m)} \rho^{l-m+s-r}\left(\frac{x-B}{2 A}\right) \\
& \times\left[1+\frac{\sqrt{t^{-1}(1-c)(1-t c)\left(1-c^{N-1}\right)}}{c^{N}\left(1-c^{-N+1}\right) \rho((x-B) / 2 A)}\right]^{l-m},
\end{aligned}
$$

where in the last step we applied the binomial theorem and where $A, B$ are as in Proposition 7.1. The case $a=l-m \leq 0$ is treated similarly; in that case develop the numerator of (A.2) using the connection formula (A.1) (so, in fact, one does the same procedure as above for the inverse of the quotient (A.2)). Let us assume from now on that $a=l-m \geq 0$. An easy calculation shows that

$$
\lim _{q \uparrow 1} r_{l, m}^{(v)}(x ; q)=R_{l, m}^{(v)}(\sqrt{1-x})
$$

with the disk polynomial $R_{l, m}^{(v)}(z)$ as in (1.1). If we now divide both sides of the addition Theorem 5.1 by the polynomial $\hat{K}_{z}\left(x q^{-N} ; t, N+m-l ; q\right)$ and make the substitutions for $q, z, N$ as before, the left-hand side will tend to

$$
(-1)^{l-m}\left[\frac{1-x}{t\left(1-c^{N}\right)}\right]^{(1 / 2)(l-m)} R_{l, m}^{(\nu)}(\sqrt{1-x})
$$


as $n \rightarrow \infty$. In the same way, using (A.3), the right-hand side tends to

$$
\begin{aligned}
& \sum_{r=0}^{l} \sum_{s=0}^{m} C_{l, m ; r, s}^{(v)}(-1)^{r-s} t^{(1 / 2)(r+s)} c^{r+s} R_{l-r, m-s}^{(v+r+s)}(\sqrt{1-t c}) \\
& \quad \times R_{l-r, m-s}^{(v+r+s)}(\sqrt{1-c}) R_{r, s}^{(v-1)}\left(\sqrt{\left.1-c^{N-1}\right)} c^{l-m}\left[\frac{1-c^{N-1}}{1-c^{N}}\right]^{(1 / 2)(l-m)}\right. \\
& \quad \times \rho^{l-m+s-r}\left(\frac{x-B}{2 A}\right)\left[1+\frac{\sqrt{t^{-1}(1-c)(1-t c)\left(1-c^{N-1}\right)}}{c^{N}\left(1-c^{-N+1}\right) \rho((x-B) / 2 A)}\right]^{l-m}
\end{aligned}
$$

with $C_{l, m ; r, s}^{(v)}$ as in (1.3). Now put $(x-B) /(2 A)=\frac{1}{2}\left(u+u^{-1}\right)$, so that $\rho((x-B) /(2 A))=$ $u$. Write

$F(\psi ; t, N ; c)=c^{l-m}\left[\frac{1-c^{N-1}}{1-c^{N}}\right]^{(1 / 2)(l-m)}\left[1+\frac{\sqrt{t^{-1}(1-c)(1-t c)\left(1-c^{N-1}\right)}}{c^{N}\left(1-c^{-N+1}\right) u}\right]^{l-m}$.

It is a straightforward exercise to verify that

$$
\begin{aligned}
(-1)^{l-m} & {\left[\frac{1-x}{t\left(1-c^{N}\right)}\right]^{(1 / 2)(l-m)} F(\psi ; t, N ; c)^{-1} } \\
& =(1-x)^{(1 / 2)(m-l)}\left(\sqrt{(1-c)(1-t c)}-u^{-1} c \sqrt{t\left(1-c^{N-1}\right)}\right)^{l-m}
\end{aligned}
$$

Moreover, from $(x-B) /(2 A)=\frac{1}{2}\left(u+u^{-1}\right)$ and from the values of $A$ and $B$ it follows that, when $z=\sqrt{(1-c)(1-t c)}-u^{-1} c \sqrt{t\left(1-c^{N-1}\right)}$ then $|z|=\sqrt{1-x}$. Now we can put all the pieces together: equate (A.4) and (A.5) and divide both sides by $F(\psi ; t, N ; c)$. Then, using (A.6) and analytic continuation to replace $u$ by $-e^{-i \psi}$, we see that we end up with

$$
\begin{aligned}
R_{l, m}^{(v)}(z)= & \sum_{r=0}^{l} \sum_{s=0}^{m} C_{l, m ; r, s}^{(v)}(-1)^{r-s} t^{(1 / 2)(r+s)} c^{r+s} R_{l-r, m-s}^{(v+r+s)}(\sqrt{1-t c}) \\
& \times R_{l-r, m-s}^{(v+r+s)}(\sqrt{1-c}) R_{r, s}^{(v-1)}\left(\sqrt{1-c^{N-1}}\right)\left(-e^{-i \psi}\right)^{s-r},
\end{aligned}
$$

where now $z=\sqrt{(1-c)(1-t c)}+c \sqrt{t\left(1-c^{N-1}\right)} e^{i \psi}$. Upon substituting $\sqrt{1-c}=$ $\cos \theta_{1}, \sqrt{1-t c}=\cos \theta_{2}, \sqrt{1-c^{N-1}}=\rho$, and invoking the homogeneity argument given in Section 7, we obtain the classical identity (1.3).

We end with a few words on the limit case $q \uparrow 1$ of the product formula (6.2). As was mentioned in Section 7 we apply [17, Theorem 2] to the orthonormal polynomials $p_{z}(x ; n)=\hat{K}_{z}\left(x c^{-N} ; t, n z N ; c^{1 / n z}\right)$. From (4.9) we derive that their orthogonality is given by

$$
\begin{aligned}
& \sum_{x=0}^{n z N} \frac{\left(c^{N} ; c^{-1 / n z}\right)_{n z N-x}\left(t c^{1 / n z} ; c^{1 / n z}\right)_{n z N-x}}{\left(c^{1 / n z} ; c^{1 / n z}\right)_{n z N-x}}\left(t c^{1 / n z}\right)^{x} \\
& \quad \times \hat{K}_{z}\left(c^{x / n z} c^{-N} ; t, n z N ; c^{1 / n z}\right) \hat{K}_{z^{\prime}}\left(c^{x / n z} c^{-N} ; t, n z N ; c^{1 / n z}\right)=\delta_{z z^{\prime}}
\end{aligned}
$$

So the measure of orthogonality for these polynomials has as support the set $\left\{c^{x / n z} \mid x=0,1, \ldots, n z N\right\}$ which is contained in the interval $[0,1]$. Again it is easy to 
check that for all $k \in \mathbb{Z}$

$$
\begin{aligned}
& \lim _{n \rightarrow \infty} \hat{a}_{n z+k}\left(t, n z N+a ; c^{1 / n z}\right)=A, \\
& \lim _{n \rightarrow \infty} \hat{b}_{n z+k}\left(t, n z N+a ; c^{1 / n z}\right)=B,
\end{aligned}
$$

with $A$ and $B$ as before. This proves that the conditions of [17, Theorem 2] are all satisfied.

Acknowledgment. Research of the second author supported by a Fellowship of the Research Council of the Katholieke Universiteit Leuven, Belgium, and by the Netherlands Organization for Scientific Research (NWO) under project number 610.06.100. We thank Tom Koornwinder for his suggestions and remarks. Part of the work of the second author was done at the Katholieke Universiteit Leuven and thanks are due to Walter Van Assche and Alfons Van Daele for their hospitality.

\section{References}

1. G. E. ANDREWS, R. ASKEY (1977): Enumeration of partitions: The role of Eulerian series and qorthogonal polynomials. In: Higher Combinatorics (M. Aigner, ed.). Dordrecht: Reidel, pp. 3-26.

2. R. ASKEY, J. A. WILSON (1979): A set of orthogonal polynomials that generalize the Racah coefficients or 6- $j$ symbols. SIAM J. Math. Anal., 10:1008-1016.

3. M. S. DiJKhUIZEN, M. Noumi (1995): A family of quantum projective spaces and related $q$ hypergeometric orthogonal polynomials. Preprint.

4. P. G. A. FloRIS (to appear): Addition formula for q-disk polynomials. Compositio Math.

5. G. GASPER, M. RAHMAN (1990): Basic hypergeometric series. In: Encyclopedia of Mathematics and its Applications, vol. 35. Cambridge: Cambridge University Press.

6. H. T. KoELINK (1991): On *-representations of the Hopf *-algebra associated with the quantum group $U_{q}(n)$. Compositio Math., 77:199-231.

7. H.T. KoELINK (to appear): Addition formulas for q-special functions. Fields Institute Comm.

8. H. T. KOELINK, R. F. SWARTTOUW (1995): A q-analogue of Graf's addition formula for the Hahn-Exton q-Bessel function. J. Approx. Theory, 81:260-273.

9. T. H. KOORNWINDER (1972): The addition formula for Jacobi polynomials, Parts II and III. Reports TW 133/72 and 135/72. Amsterdam: Math. Centrum.

10. T. H. KOORNWINDER (1991): The addition formula for little q-Legendre polynomials and the SU(2) quantum group. SIAM J. Math. Anal., 22:295-301.

11. T. H. KOORNWINDER (1993): Askey-Wilson polynomials as zonal spherical functions on the SU(2) quantum group. SIAM J. Math. Anal., 24:795-813.

12. M. NOUMI, K. MiMACHI (1990): Askey-Wilson polynomials and the quantum group $S U_{q}$ (2). Proc. Japan Acad. Ser. A Math. Sci., 66:146-149.

13. M. NOUMI, H. YAMADA, K. MiMACHI (1993): Finite dimensional representations of the quantum group $G L_{q}(n ; C)$ and the zonal spherical functions on $U_{q}(n-1) \backslash U_{q}(n)$. Japanese J. Math., 19:31-80.

14. R. L. S̆APIRO (1993): Special functions connected with representations of the group $S U(n)$ of class I relative to $S U(n-1)(n \geq 3)$. Izv. Vysš. Učebn. Zaved. Mathematika, 71:9-20 (Russian); AMS Translation Series 2, 113:201-211 (English).

15. D. STANTON (1984): Orthogonal polynomials and Chevalley groups. In Special Functions: Group Theoretical Aspects and Applications (R. A. Askey, T. H. Koornwinder, W. Schempp, eds.). Dordrecht: Reidel, pp. 87-128.

16. L. L. VAKSMAN, Y. S. SOIBELMAN (1988): Algebra of functions on the quantum group SU(2). Funct. Anal. Appl., 22:170-181.

17. W. VAN ASSCHE, T. H. KoORNWINDER (1991): Asymptotic behaviour for Wall polynomials and the addition formula for little q-Legendre polynomials. SIAM J. Math. Anal., 22:302-311. 
18. N. J. VILENKIN (1968): Special Functions and the Theory of Group Representations. Transl. Math. Monographs, vol. 22. Providence, RI: American Mathematical Society.

19. N. J. Vilenkin, A. U. KLIMYK (1991)-(1993): Representation of Lie groups and Special Functions, 3 volumes. Dordrecht: Kluwer Academic.

P. G. A. Floris

Afdeling Wiskunde en Informatica

Rijksuniversiteit Leiden

P.O. Box 9512

2300 RA Leiden

The Netherlands

floris@wi.leidenuniv.nl
H. T. Koelink

Vakgroep Wiskunde

Universiteit van Amsterdam

Plantage Muidergracht 24

1018 TV Amsterdam

The Netherlands

koelink@fwi.uva.nl 